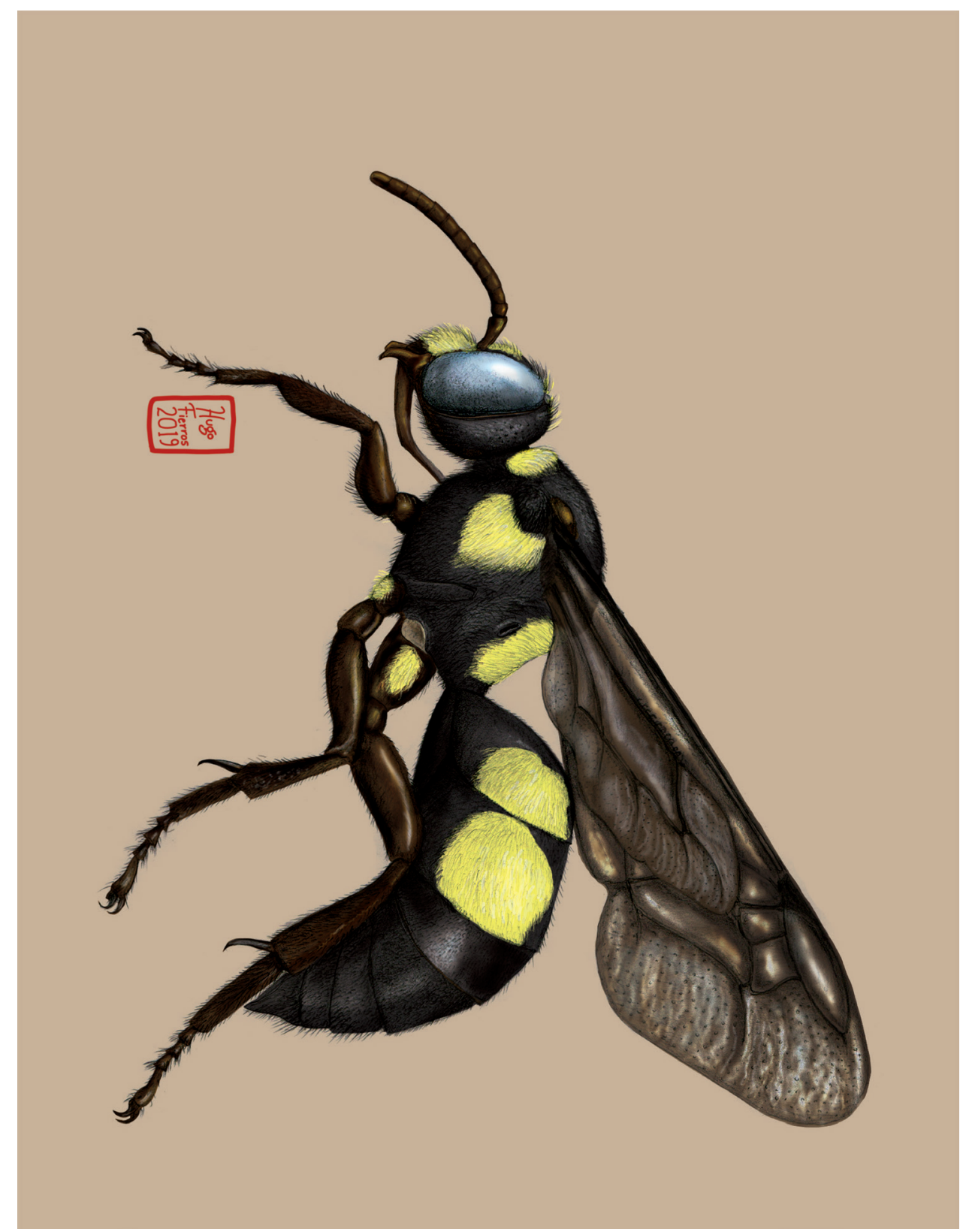

Dugesiana, Año 27, No. 1, enero 2020-junio 2020 (primer semestre de 2020), es una publicación semestral, editada por la Universidad de Guadalajara, a través del Centro de Estudios en Zoología, por el Centro Universitario de Ciencias Biológicas y Agropecuarias. Camino Ramón Padilla Sánchez \# 2100, Nextipac, Zapopan, Jalisco, Tel. 37771150 ext. 33218, http://148.202.248.171/dugesiana/index.php/DUG/index, glenusmx@gmail.com. Editor responsable: José Luis Navarrete-Heredia. Reserva de Derechos al Uso Exclusivo 04-2009-062310115100-203, ISSN: 2007-9133, otorgados por el Instituto Nacional del Derecho de Autor. Responsable de la última actualización de este número: José Luis Navarrete-Heredia, Editor y Ana Laura González-Hernández, Asistente Editorial. Fecha de la última modificación 1 de enero 2020, con un tiraje de un ejemplar.

Las opiniones expresadas por los autores no necesariamente reflejan la postura del editor de la publicación.

Queda estrictamente prohibida la reproducción total o parcial de los contenidos e imágenes de la publicación sin previa autorización de la Universidad de Guadalajara. 
Artículo

\title{
Los Platipodinos (Coleoptera: Curculionidae: Platypodinae) del estado de Morelos, México
}

\author{
Platyipodines (Coleoptera: Curculionidae: Platypodinae) from Morelos State, Mexico
}

\author{
Armando Burgos Solorio ${ }^{1}$ y Norberto Hernández ${ }^{2}$ \\ Laboratorio de Parasitología Vegetal ${ }^{1}$, Maestría en Manejo de Recursos Naturales ${ }^{2}$ Centro de Investigaciones \\ Biológicas, Universidad Autónoma del Estado de Morelos, Cuernavaca, Morelos, México.burgos@uaem.mx
}

\section{RESUMEN}

Derivado de información publicada y colectas de escarabajos ambrosiales de la subfamilia Platypodinae (Curculionidae), realizada en los diferentes tipos de vegetación inmersos en el estado de Morelos en la que se registran un total de nueve especies incluidas en cinco géneros, siendo Euplatypus con cinco especies. Se presenta una lista comentada sobre biología, plantas asociadas, distribución, y se adicionan nuevos registros como por ejemplo Oxoplatypus quadridentatus, y hace mención de entre otra información. Se reclasifica Platypus exisus por Teloplatypus ustulatus. Se anexa una clave taxonómica de las especies y se incorporan imágenes mostrando los caracteres morfológicos distintivos de los taxones distribuidos en la entidad.

Palabras clave: Escarabajos ambrosiales, diversidad, Eje Neovolcánico Transversal, Cuenca del Balsas, plantas hospederas.

\section{ABSTRACT}

Derived from published information and collections of ambrosial beetles of the subfamily Platypodinae (Curculionidae), carried out in the different types of vegetation immersed in the state of Morelos in which a total of nine species included in five genera are registered, Euplatypus being five species. A commented list is presented on biology, associated plants, distribution, and new records are added, such as Oxoplatypus quadridentatus, and mention among other information. Platypus exisus is reclassified by Teloplatypus ustulatus. A taxonomic key of the species is attached and images are incorporated showing the distinctive morphological characters of the taxa distributed in the state.

Key words: Ambrosial beetles, diversity, Transvolcanic Belt, Balsas Basin, host plants.

La subfamilia de Platypodinae cuyo género tipo "Platypus" deriva del griego "Platypous", que significa "Patas planas" son conocidos como "escarabajos "perforadores" o ambrosiales" de importancia en los ecosistemas vegetales que van desde las regiones holárticas, desérticas hasta las tropicales y subtropicales del mundo (Wood, 1982; Burgos y Equihua, 2007).

Un carácter distintivo del grupo es presentar un mismo tipo de habito alimentario, al aprovechar los tejidos y fibras de las diferentes partes anatómicas de las plantas leñosas como árboles y arbustos de diferentes familias taxonómicas, asimismo otra característica importantes que deriva de su nombre común "ambrosia" que significa "El alimento de los dioses" es la simbiosis que guardan entre hongos y escarabajos, ejemplo de ello son Fusarium solani, F. oxyporum, Alternaria alternata y Botryodiplodia theobromae entre otros, que provocan muerte de árboles de importancia forestal y económica en cultivares del nogal pecanero del norte de México [Carya illinoinensis (Wangenh.)], o en aquellas especies como Pithecellobium dulce, Maguifera indica y plantas ornamentales como Delonix regia, Ficus retusa, Fraxinus udhei entre otras (Alvidrez-Villarreal, 2010; Samaniego-Gaxiola, et. al. 2008; Atkinson et. al. 1986b).

Las referencias sobre los "daños" ocasionados por estos barrenadores es limitada, estos actúan cortando los tejidos encargados del transporte de agua y nutrientes e inciden en los tejidos de sostén de las plantas, lo que trae consigo el marchitamiento y muerte de los mismos. Otro aspecto importante es la relación que guardan entre escarabajos ambrosiales y hongos; estos insectos actúan como agentes etiológicos al almacenar esporas en estructuras especializada llamada micangios. Estos hongos simbiontes principalmente del orden Ophiostomatales (Ascomycota, Sordariomycetes) son un grupo polifilético de especies, particularmente adaptados a la dispersión por escarabajos de la corteza. Su importancia radica en que muchas especies son patógenas y otras provocan el deterioro de la celulosa, lignina y sustancias como el almidón, cuyos peritecios lo utilizan como sustrato y a la vez provocan el manchado "azul de la madera", síntoma que reduce su valor comercial; por el contrario la relación simbiótica que existe entre los hongos y barrenadores es compleja y transcendente, es decir por una parte los hongos forman parte de la dieta alimenticia de larvas y adultos de estos escarabajos y por el otro están los hongos que no producen pudrición de la madera como los que manchan la superficie, como las especies del género Ceratocistis entre otros, que utilizan como alimento los almidones, azúcares, proteínas y/o grasas almacenadas, lo que produce decoloraciones, pero tienen poco efecto en la dureza de la madera; asimismo escarabajos y hongos simbióticos, aceleran la descomposición de la misma, es decir, la acción de los escarabajos barrenadores al establecerse, construir la galería y desarrollarse y diseminar los hongos, aprovechan los tejidos de la planta como la celulosa y lignina y sustancias como azúcares, almidones, proteínas y grasas; todo ello trae como consecuencia el proceso de descomposición 
de los tejidos y el reciclaje de nutrientes en los ecosistemas vegetales. Lo antes mencionado revela que la función ecológica de hongos e insectos es preponderante al sumar sus funciones en la compleja pero importante ecología de los ecosistemas vegetales (Batra, 1985 y 1966; Freeman et. al., 2013; Alonso et. al., 2014, Eskalen et. al., 2014).

La subfamilia Platypodinae está integrada por 1, 376 especies incluidas en 38 géneros a nivel mundial, de éstas 43 especies pertenecientes a 11 géneros se encuentran distribuidos en los diferentes ecosistemas vegetales de México (Atkinson, 2019). Acorde a Burgos y Trejo, (2001) y Atkinson (2019) para Morelos el grupo lo integran siete especies, de los géneros Euplatypus con cuatro y Schedlarius, Megaplatypus y Teloplatypus con una especie respectivamente.

La mayoría de las especies del género Euplatypus presentan una distribución neotropical, excepto para Schedlarius endémico de Centroamérica, en selvas secas, Euplatypus parallelus con distribución pantropical y Oxoplatypus que presenta una distribución holártica; para los géneros de Megaplatypus y Teloplatypus su distribución va desde México hasta Argentina (Wood, 1993). Los trabajos sistematizados o colectas esporádicas en México sobre el grupo, abordan aspectos faunísticos y distribución de algunas regiones del país como la Estación Biológica Chamela, Jalisco, región norte de Morelos, Cañón de Lobos, Sierra de Huautla en Morelos, Villa Hermosa, Tabasco; derivado de ello se ha integrado información biológica (planta hospedera), taxonómica, ecológica entre otros datos que conducen al conocimiento básico del grupo pero a la vez a la dinámica ecológica de bosques y selvas (Schedl, 1940; Equihua et. al., 1984; Equihua, 1985; Atkinson y Equihua, 1985; Atkinson y Equihua, 1986; Equihua y Atkinson, 1987; Burgos y Equihua, 2007).

Con el objeto de conocer más de este taxa, se estudiaron 158 ejemplares que pertenecen a nueve especies de los géneros Euplatypus (cinco especies), Schedlarius, Megaplatypus, Teloplatypus y Oxoplatypus con una especie respectivamente. Se integra y actualizan las sinonimias de cada taxón, así como su diagnosis, distribución en el estado, rango altitudinal, tipo de vegetación, habito, especificidad, hospederos, e imágenes de los hábitos de macho-hembra y observaciones específicas, y se presenta un resumen de las especies de platipodinos registrados para Morelos. Los datos sobre la distribución de especies por tipo de vegetación mostraron un patrón claro de la distribución de los platipodínos, ya que la selva baja caducifolia alberga más del 50\%, seguida del bosque de Pinus-Quercus, mesófilo de montaña y agricultura perene y ornamental con $25 \%$ y bosque de Abies-Pinus con un $12.5 \%$. Cabe aclarar que hay especies que pueden traslaparse de una vegetación a otra como por ejemplo Euplatypus pini el cual se asocia a bosques de Abies-pino y pino encino, así como Euplatypus parallelus y E. segnis asociado a zonas de transición entre bosques de pino-encino y selva baja caducifolia. Se generó una clave taxonómica con los ejemplares revisados para el reconocimiento morfológico e imágenes para complementar su reconocimiento entre otra información inherente a este grupo taxonómico

\section{MATERIALES Y MÉTODOS \\ Zona de estudio}

Morelos se localiza en la parte Centro-Sur de la República Mexicana. Cuenta con una superficie 4, $879 \mathrm{~km}^{2}$ se ubica la vertiente sur del Eje Volcánico Transversal, forma parte de la Cuenca del río Balsas y está limitado al sur por la Sierra Madre del Sur y montañas de la Mixteca en Oaxaca. Está localizado entre los paralelos $18^{\circ} 22^{\prime} 06^{\prime \prime}$ y $19^{\circ} 07^{\prime} 10^{\prime \prime}$ de latitud norte y entre los meridianos $98^{\circ} 08^{\prime} 06^{\prime \prime}$ y $99^{\circ} 07^{\prime} 10^{\prime \prime}$ de longitud oeste. En la zona central se encuentra la Sierra de Yautepec, que corre de norte a sur, al igual que la Sierra de Tlaltizapan; estas dos conformaciones montañosas limitan valles y llanuras, que hacen a la región tener hábitats muy particulares en condiciones climáticas y orográficas, situados a una altitud que van de los 5, $452 \mathrm{~m}$ localizado en las faldas del Popocatépetl a los $780 \mathrm{~m}$ en la localidad de Nexpa; asimismo destacan los seis tipos de vegetación entre los que sobresalen el bosque de pino-encino y la selva baja caducifolia, lo que hace de la región un sitio idóneo para el establecimiento de este grupo de escarabajos (Anónimo, 1981; Aguilar, 1990; Dorado, 1997).

El documento está basado en información bibliográfica, además de recolectas realizada por los autores; asimismo se complementa y se integra información de ejemplares resguardados en las siguientes colecciones:

- Colección entomológica (CEUM), Laboratorio de Parasitología Vegetal, CIB-UAEM

- Colección Entomológica del Laboratorio de Entomología, Centro de Investigaciones Biológicas, UAEM

- Colección del Instituto de Fitosanidad del Colegio de Posgraduados, Montecillos (CEAM).

Se tomaron imágenes de los escarabajos adultos (macho y hembra) en las que se muestran los principales caracteres morfológicos de las especies, tomadas con un microscopio estereoscópico modelo SMZ 1500 y una cámara digital DXM 1200C Nikon. Las imágenes se procesaron con el programa Combine ZM y Adobe PhotoShop versión 7.0 y se utilizó el programa Image Tool 3.0 para realizar las mediciones correspondientes del material analizado, así como mostrar las principales estructuras morfológicas distintivas de los taxones. Asimismo, se hace mención de la distribución estatal de las especies de escarabajos ambrosiales basada en las colectas realizadas por los autores. Finalmente se actualiza la información de los taxones a nivel específico acorde al criterio de Wood (1993).

\section{RESULTADOS}

Derivado de este estudio se analizaron más de 158 ejemplares, que pertenece a nueve especies, incluidas en los géneros Euplatypus (cinco especies), Schedlarius, Megaplatypus, Teloplatypus y Oxoplatypus con una especie por cada género. Megaplatypus exaratus y Oxoplatypus quadridentatus constituyen un nuevo registro para la entidad ampliándose el rango de distribución para el país (Cuadro 1).

Se integra información sobre biología, plantas hospe- 
deras, distribución altitudinal, georreferenciación y observaciones en general, se actualizan los nombres genéricos y específicos, acorde a la revisión genérica de la subfamilia (Wood, 1993), como es el caso de las especies de Euplatypus, Teloplatypus ustulatus y Megaplatypus exaratus, la cual se redescribió recientemente, se consideran los caracteres específicos de la especie Oxoplatypus quadridentatus considerados en la clave taxonómica (Blackman, 1922. Burgos y Atkinson, 2015), Asimismo género y especie constituyen un nuevo registro para la entidad.

El material colectado de este grupo, permite proporcionar una clave para la identificación e información acerca de la biología de las especies basada en ejemplares adultos de acuerdo al criterio de Wood, 1992 y 1993, para la identificación y el reconocimiento de las especies de Platipodinos. Esta clave es válida para las muestras colectadas en la entidad. El arreglo taxonómico sigue el criterio de los autores antes mencionados.

\section{Clave taxonómica de las especies de Platipodínos del estado de Morelos}

Debido a que el grupo presenta un dimorfismo sexual marcado, en la clave taxonómica solo se consideran características morfológicas del macho, los cuales se distinguen de las hembras, por presentar una serie de espinas o proyecciones en la parte anterior de los élitros excepto en Schedlarius en donde los adultos son muy parecidos morfológicamente. El criterio de la clave está basado en Wood (1993) y modificadas por los autores

1.- Escarabajos de cuerpo cilíndrico y alargado; cabeza tan ancha como el pronoto masa antenal sin suturas (excepto el género Coptonotus); esternón abdominal armado con espinas; primer segmento tarsal tan largo como el 2 y 5 combinados, cortos en algunos géneros; mucro apical sobre la protibia formada primitivamente desde un proceso apical medio; dentículos laterales de la protibia, nunca en punta; especies con la presencia o ausencia de poros micangiales; de hábitos xilomicetófagos y xilófagos de talla variable ... (PLATYPODINAE) ...................... 2 1'.- Margen posterior del protórax ligeramente precurvado en área epimeral, mesopisternum moderadamente largo y plano; mesostergum plano y ancho en vista lateral, ligeramente arqueado, sin una carina media; Escutelo desarrollado; cabeza con la pregula pequeña en vista ventral, con una carina transversa; ojos prominentes; escapo delgado, mazo antenal cortado en su extremo anterior con 5 segmentos; procoxa pequeña; primer segmento tarsal largo, sutura anapleural sobre el mesotórax presente; especies xilófagas. Protibia larga, armada con espinas, con 3 segmentos tarsales, anchos y bilobados; élitros con el declive oblicuo, subtruncado y ornamentado entre las interestrías por escamas blancas y armando en su margen por una hilera de espinas, sobre Bursera, de México a Nicaragua (Figura 2: a - f) ............ Schedlarius mexicanus

2.- Esternón abdominal del macho armado por espinas o dentículos en el borde anterior del macho, de talla variable... 3 $2^{\prime}$.- Esternón abdominal presente en el 4 segmento del ma- cho con un diente agudo sobre cada lado de la línea media de los élitros, talla menos a $4.5 \mathrm{~mm}$ (Figura 4: a-d) ....... Oxoplatypus quadridentatus

3 (2).- Especies mayores de $7 \mathrm{~mm}$ (excepto Teloplatypus)...4 3'.- Especies menores a $7 \mathrm{~m}$........................................ 5

4 (3).- Macho con el declive elitral con cinco hileras estriales bien marcadas y ornamentadas con una serie de proyecciones o dentículos que no se extienden más allá del margen posterior; Poros micangiales sobre el pronoto, poco común en hembra y raro en macho; Especies de una talla de 2.3 a 10 mm (Figura 6: a-d) ..................... Megaplatypus exaratus 4".- Declive del macho aserrado en el borde los élitros en la región posterior en vista lateral, con una carina dorsal que se extiende de espina a espina sobre la interestría en la base del declive, margen basal del ápice usualmente armado por pequeñas espinas sobre las interestría 1,3,5; poros micangiales ausentes; de México a Argentina; 2.2 -4.2 mm sobre leguminosas. (Figura 8: a-d) ................ Teloplatypus ustulatus

5 (3').- Especies con los poros micangiales ausentes en ambos sexos, de distribución tropical altamente polífaga ...... 6 $5^{\prime}$.- Especies con los poros micangiales en hembras $o$ en ambos sexos, distribuida en climas templados y fríos, asociadas a gimnospermas y angiospermas latifoliadas

6 (5).- Declive elitral angosto hacia la región apical en vista dorsal, con procesos ventrolaterales proyectados en puntas bien definidas, cada proceso armados con tres dientes bien definidos; $3.6 \mathrm{~mm}$ de longitud (Figura 10: a-d) ......................................... Euplatypus segnis 6'.- Declive elitral más ancho hacia la región apical en vista dorsal, con procesos ventrolaterales proyectados en punta no tan marcadas (roma), cada proceso armado con tres dientes en forma de gránulos; $4.1 \mathrm{~mm}$ de longitud (Figura 12: a-d) ......................... Euplatypus parallelus

$7\left(5^{\prime}\right)$.- Declive de macho ligeramente pronunciado antes del ápice; con cuatro líneas, la primera (a partir de la línea media de los élitros) poco marcada; la segunda fuertemente abultada y marcada, las dos restantes evidentes; todas armadas con dientes en forma de ángulo (excepto la primera); margen apical con dos proyecciones bífidas, agudas en forma de "V"; solo en especies de pinos. (Figura 14: a-d) ........................... Euplatypus pini $7^{\prime}$.- Especies con dos proyecciones agudas en el borde posterior de los élitros, asociados a latifoliadas ........................ 8

8 ( $\left.7^{\prime}\right)$.- Declive del macho con seis hileras poco marcadas, excepto las dos laterales a partir de la línea media y ornamentadas con una hilera de sedas doradas, localizadas en su declive; borde laterales angostos y aserrados; borde apical con dos proyecciones agudas, sobre $\mathrm{Al}$ nus. (Figura 16: a-d) .................... Euplatypus longius $8^{\prime}$ Macho con 3 hileras, no muy marcadas excepto la primera y prominente, armadas con unas proyecciones agudas; con 4 interestrías ligeramente evidentes. En el borde posterior en 
forma de "U" y con dos proyecciones con los bordes laterales aserrados y con las puntas bífidas; sobre especies de Quercus y Alnus (Figura 18: a-d) Euplatypus otiosus

\section{Lista comentada de las especies de Platypodinae del estado de Morelos}

Curculionidae Latreille, 1802

Platypodinae Shuckard, 1840

Schedlariini Wood, 1993

Schedlarius Wood, 1993

(=Chapuisia)

Schedlarius mexicanus (Dugès, 1885)

(Figuras 1-2)

Chapuisia mexicana (Dugès, 1885) Ann. Soc. Ent. Belq. 29(2): 56 Schedlarius mexicanus (Dugès, 1885) Ann. Soc. Ent. Belq. 29(2): 56

Diagnosis. Esta especie se distingue del resto de los platipodinos por presentar un cuerpo robusto, alargado y cilíndrico; talla de $2.3 \mathrm{~mm}$, color café oscuro, cuerpo ornamentado con escamas blancas en regiones del cuerpo. Quetotaxia en forma de escamas, éstas compuestas por sedas finas en forma de plumón, distribuidas en la región del borde posterior de los ojos, región epipleural del pronoto, epipleura, episternum, metasternum, y externitos y en la región anterior del borde los élitros de limitada por el callus humeral, parte lateral-media y el declive de los élitros (Figuras 1-6). Élitros de talla de un $1.4 \mathrm{~mm}$, más angostos en la región del borde anterior, el declive abarca $1 / 3$ de los élitros, delimitado por una hilera de dientes prominentes y agudos en la región anterior del declive, cabeza expuesta y delimitada por el borde anterior del pronoto.

Distribución. Costa Rica a México. México: Chiapas, Guanajuato, Guerrero, Jalisco, Morelos, Oaxaca, Puebla (Atkinson, 2019).

Rango altitudinal. 990-1780 msnm.

Tipo de Vegetación. Selva baja caducifolia.

Hábito. Xilófago.

Especificidad. Monófago.

Material examinado. México; Morelos, TEPOZTLÁN, Amatlán, 1780 m, Bursera, sp., SM-331; Sta. Catarina, 1610 m, Bursera copallifera (1); 1720 m, B. copallifera, S-536(2); El Texcal, $1710 \mathrm{~m}$ Bursera sp. AB84; YAUTEPEC: Cañón de Lobos, 1440 m, Bursera sp., SM-162 (2); 1360 m, B. fagaroides, SM-184(1); 1, 320 m, Bursera sp. SM-201; 1460 m, Bursera sp., Sm-228; TLALTIZAPAN: Nueva Temilpa, 990 m, Bursera sp. S-584.; Tlaquiltenango Carr. Quilamula-Huautla km 15, 12-04-1997, 1020 m, Bursera sp. (2), Cols. Equihua-Burgos; Huautla-Huaxtla, 15-04-2000, $18^{\circ} 26^{\prime} 10^{\prime \prime} \mathrm{N} 99^{\circ} 01^{\prime} 25^{\prime}$ ' W, 1350 $\mathrm{m}$, colectado al vuelo, Col. J. Romero (1); PUENTE DE IXTLA, Cerro el Jumilar, 16-02-2002, $1250 \mathrm{~m} 18^{\circ} 20^{\prime} 53^{\prime \prime} \mathrm{N}$ 99¹4' 94'W, Bursera sp, A. Burgos (4).

Hospederos. Burseraceae: Bursera copallifera, Bursera fagaroides, Bursera simaruba y Bursera sp. (Atkinson, 2019).

Observaciones. Este escarabajo infesta a troncos y ra- mas de un diámetro entre los 10 hasta $30 \mathrm{~cm}$ de ramas y árboles derribados; por lo general las entradas de las galerías se localizan en las partes laterales, evadiendo la luz directa del sol, y evitando de esta manera un sobrecalentamiento en el interior de la galería.

Se capturó un ejemplar en vuelo, en el crepúsculo; este hábito es típico en este grupo de escarabajos, pero poco frecuente colectarlos de esta manera. El insecto infesta e inicia la galería preferentemente en ramas y troncos en donde quede expuesta la madera o en heridas provocadas de manera intencional o en ramas rotas por la acción del viento. En unas muestras confinadas después de dos o tres meses se le ha observado actividad, lo que nos indica que se encuentran generaciones traslapadas en las muestras analizadas. En una muestra colectada en el cerro el "Jumilar" sobre Bursera (posiblemente de la especie copallifera), este barrenador forma su galería, con entrada principal y después de 1 a $2 \mathrm{~cm}$, se bifurca en direcciones opuestas, mismas que penetra hasta el xilema del huésped. Larvas y pupas son blanquecinas, hialinas.

\section{Platypodini \\ Platypodina \\ Oxoplatypus Wood, 1993 \\ Oxoplatypus quadridentatus (Olivier) 1795 \\ (Figuras 3-4)}

Oxoplatypus quadridentatus (Olivier, 1795)

Platypus quadridentatus (Olivier, 1795)

Scolytus quadridentatus Olivier, 1795.

Platypus blanchardi Chapuis, 1865.

Platypus disciporus Chapuis, 1865.

Platypus flavicornis disciporus LeConte, 1876.

Diagnosis: Macho color café obscuro Talla $4.48 \mathrm{~mm}$ de longitud.

Distribución: Norte América: Estados unidos, México. En México únicamente se han realizado tres colectas una por estado en Chihuahua, Nayarit y Nuevo León (Atkinson, 2019).

Rango altitudinal: 1792 a $1917 \mathrm{msnm}$.

Tipo de vegetación: Asociada a bosque de encino, encontrándose en la parte norte del estado en zonas frías.

Hábito: Xilomicetófago.

Especificidad: Polífago.

Material examinado: Morelos: CUERNAVACA, Buenavista del Monte, 21-02-2018, 1855 msnm, NH-322, Quercus magnoliifolia Née., Cols. N. Hernández y A. Burgos, (5 0 y 6 우); 21-02-2018, 1792 msnm, NH323, Quercus magnoliifolia Née., Cols. N. Hernández y A.

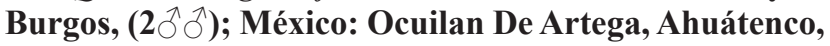
07-02-2018, 1917 msnm, NH-316, Quercus magnoliifolia

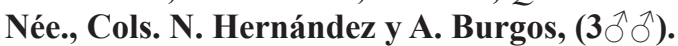

Hospedero: Se tiene como plantas hospedaras a las siguientes especies: Fagaceae: Castanea dentata, Castanea sp.; Fagus sp.; Quercus affinis, Quercus alba, Quercus borealis, Quercus coccinea, Quercus hemisphaerica, Quercus laurifolia, Quercus marylandica, Quercus montana, Quercus nigra, Quercus rubra, Quercus sp., Quercus velutina, 
Quercus virginiana; Hamamelidaceae: Liquidambar styraciflua; Hippocastanaceae: Aesculus octandra; Pinaceae: Pinus palustris; Tsuga canadensis. (Atkinson, 2019).

Observaciones. Se encuentra en zonas frías (holártica). Se encuentra en árboles de encino derribados, cubiertos por hojarasca y vegetación con humedad, en troncos de entre 60 a 80 centímetros de diámetro. Se distinguen las entradas de las galerías por la presencia de abundante debris en forma de polvo o en proyecciones cilíndricas en forma de churros de un centímetro expuestos fuera del tronco, así como por la madera manchada de color negro milímetros más allá de la circunferencia de la galería (posiblemente por la presencia del hongo); se encuentran en toda la superficie de la base, siendo más abundantes en donde hay sombra en el tronco, evitando los rayos directos del sol. Las entradas se forman de tres a cuatro centímetros desde la corteza al interior del tronco en línea recta donde se bifurca en direcciones opuestas en los límites del xilema, posteriormente se muestran difusas entrecruzándose en la mayoría de los casos; es en el xilema donde se desarrollan las galerías y donde se pueden encontrar una mayor cantidad de insectos y de ambos sexos ya que por lo regular en las entradas solo se logran encontrar los machos limpiando las entradas de las galerías.

La colecta de esta especie constituye un nuevo registro para la entidad, lo que revela el registro más sureño sobre la distribución de la especie. La forma de la galería es sinuosa a irregular dirigida al xilema de la planta. La especie de planta a la que se asocia este barrenador (Quercus magnoliifolia Née.) constituye un nuevo registro.

\section{Megaplatypus Wood, 1993 Megaplatypus exaratus (Blandford, 1895)} (Figuras 5-6)

Megaplatypus exaratus (Blandford 1895), Biol. Cent. Amer. 4(6): 98 Platypus exaratus (Blandford, 1895), Biol. Cent. Amer. 4(6): 98

Diagnosis. Macho color café claro a tonalidades oscuras; Talla 5.83 a $7.8 \mathrm{~mm} \pm 1.95 \mathrm{~mm}$ de longitud. La hembra $7.4 \mathrm{~mm}$, (Figuras 7-13).

Distribución. América central: Costa Rica, Guatemala; Norte América: México: en el país se registran en Veracruz, Xalapa; Oaxaca, Oaxaca, Estado de México, Chiapas, Morelos: Yautepec, Tepoztlán, (Atkinson y Equihua, 1986, Atkinson et. al., 1986a y b; Noguera y Atkinson, 1990, Atkinson, 2019).

Rango altitudinal. 1360 a los $1542 \mathrm{msnm}$, presumiblemente esta especie puede presentar un rango de distribución más amplio, por lo que es necesario confirmar este dato para ratificar su distribución altitudinal.

Tipo de vegetación. Selva baja caducifolia, Vegetación ornamental perennifolia.

Hábito. Xilomicetófago.

Especificidad. Polífago.

Material examinado. Morelos: YAUTEPEC, Cañón de Lobos, 11-11-1983, 1360 m, SM-202 Erythrina breviflora, cols. E. Martínez y A. Burgos, (4ठํ ), 11-111983, 1360 m, SM-202 (2 ठ̊ đ̊) Col. E. Martínez, No. Cat. 313, Erythrina sp. (2 ふَ̋); 20-11-1983, No. Cat.
314, Ficus cotinifolia $(12$ ふぇ) Col. E. Martínez, 1380 m, SM-196 (1); 1360 m, Erythrina brevifolia, ESM202; 1460 m, Ficus cotinifolia, SM-207, TEPOZTLÁN, Santiago Tepetlapa, 23-06-1983, No. Cat. 144 Cols. E. Martínez y E. Saucedo (1ठ̂). Tepoztlán: Santiago Tepetlapa, 1600 m, SM-315.

Hospedero. Moraceae: Ficus cotinifolia; Ficus insípida, Pseudolmedia spuria; Malvaceae: Heliocarpus appendiculatus; Fabaceae: Erythrina breviflora, Inga sp.; Papilionaceae: Erythrina brevifolia, Taxodiaceae: Taxodium mucronatum. (Martínez, 1984; Atkinson y Equihua, 1986 y Atkinson et. al., 1986a y b; Noguera y Atkinson, 1990, Atkinson, 2019).

Observaciones. Esta especie es xilomicetófaga, monógama y polífaga (Noguera y Atkinson, 1990). Este escarabajo fue colectado en troncos gruesos de una leguminosa comúnmente conocida como "colorín", de aproximadamente $90 \mathrm{~cm}$ de diámetro, de árboles talados: la galería es construida en las partes laterales del fuste, esto permite evadir la luz directa del sol, lo que trae por consecuencia que la temperatura aumente, evitando de esta manera que el escarabajo se establezca en esta parte del hospedero. Por lo general se establece en lugares húmedos cerca de arroyos, situación que prevalece en la zona conocida como Cañón de Lobos, Yautepec, Morelos (Martínez, 1984; Atkinson et. al., 1986b). Especie poco común, se limita a arroyos húmedos.

\section{Teloplatypus Wood, 1993 Teloplatypus ustulatus (Chapuis, 1865)} (Figuras 7-8)

Platypus obsoletus Chapuis ,1865 Monographie des Platypides: 228 Platypus petersi Chapuis, 1865 Monographie des Platypides: 226 Teloplatypus ustulatus (Chapuis, 1865) Monographie des Platypides: 224 Platypus ustulatus Chapuis, 1865 Monographie des Platypides: 224

Distribución. América central: El salvador, Costa Rica, Guatemala, Honduras, Panamá; El Caribe: República Dominica, Jamaica; América del sur: Brasil, Colombia, Guayana Francesa, Perú, Venezuela; Norte América: México. Para México, las colectas se registran en Chiapas, Guerrero, Jalisco, Nayarit, Oaxaca, San Luís Potosí, Tamaulipas, Veracruz (Atkinson, 2019).

Rango altitudinal. 780 a los $1120 \mathrm{msnm}$.

Tipo de vegetación. - Selva baja caducifolia.

Hábito. Xilomicetófago.

Especificidad. Oligófago.

Material examinado. México; Morelos, TEPALCINGO: Los Sauces, $1400 \mathrm{~m}, \mathrm{AB}-162$ (2); TLAQUILTENANGO: El Vergel, $920 \mathrm{~m}$, Pithecellobium dulce, SM-96 (2), AB-124 (2); XOCHITEPEC: Atlacholoaya, $1120 \mathrm{~m}$, $P$. dulce SH-353; PUENTE DE IXTLA, Los Estanques-La Bejuquera, 29-09-2001, 1000 m, Lysiloma sp., Col. A. Burgos (1); TLAQUILTENANGO: Nexpa, 31-011999, 780 m, 18 $31^{\prime} 03^{\prime \prime}$ W 99 $08^{\prime} 06^{\prime \prime} \mathrm{N}$, Pithecellobium dulce, Col. A. Burgos (20), El Vergel, 920 m, 03-12-1982, Pithecellobium dulce, Col. Burgos y Saucedo, SM-96 (2).

Hospederos. Fabaceae: Acacia sp., Enterolobium cyclocarpum, Erythrina costaricensis; Inga sp.; Loncho- 
carpus margaritensis; Moraceae: Cecropia sp. (Atkinson, 2019); Mimosaceae: Pithecellobium dulce.

Observaciones. Este barrenador presenta el patrón de comportamiento típico del género. Infesta troncos de árboles de un diámetro de 10 a $15 \mathrm{~cm}$ hasta troncos de un diámetro de $50 \mathrm{~cm}$, por lo general de árboles derribados en proceso de fermentación. Se ha observado que la distribución de este escarabajo se limita principalmente a las partes tropicales, penetrando en regiones subtropicales (zonas de transición) de la zona de estudio, localizadas al sur de la entidad. En algunos casos se le ha encontrado asociado con Xyleborus volvulus en las inmediaciones de Quilamula, Tlaquiltenango. Cabe aclarar que esta especie fue determinada erróneamente a nivel genérico y específico como Platypus, Euplatypus exisus (Atkinson, et. al., 1986b; Burgos y Trejo, 2001), sin embargo, acorde a Wood (1993) y Atkinson (2019), la especie se reubica como Teloplatypus ustulatus.

\section{Euplatypus Wood, 1993 Euplatypus segnis (Chapuis, 1865)}

(Figuras. 9-10)

Platypus apertus Chapuis, 1865. Monographie des Platypides: 169 Euplatypus hians (Chapuis, 1865), Monographie des Platypides: 167 Platypus hians Chapuis, 1865. Monographie des Platypides: 167 Platypus melanurus Chapuis, 1865. Monographie des Platypides: 180 Platypus perpusillus Chapuis, 1865. Monographie des Platypides: 171 Platypus poriferus Chapuis, 1865. Monographie des Platypides: 168 Euplatypus pulicaris (Chapuis, 1865) Monographie des Platypides: 165 Platypus pulicaris Chapuis, 1865. Monographie des Platypides: 165 Platypus schaumi Chapuis, 1865. Monographie des Platypides: 181 Euplatypus segnis (Chapuis, 1865) Monographie des Platypides: 166 Platypus segnis Chapuis, 1865. Monographie des Platypides: 166 Platypus viduus Chapuis, 1865. Monographie des Platypides: 178 Euplatypus bellus (Schedl, 1933) Schedl, 1933c: 172

Platypus bellus Schedl, 1933. Rev. de Ent. 3: 172

Platypus virgatus Schedl, 1935. Rev. de Ent. 5: 355

Euplatypus costaricensis (Schedl, 1936) Arch. Inst. Biol. Veg. Rio de Janeiro 3: 101

Platypus costaricensis Schedl, 1936. Arch. Inst. Biol. Veg. Rio de Janeiro 3: 101

Platypus nitidipennis Schedl, 1949. Not. Mus. de la Plata Zool. 14(116): 41

Distribución. América Central: Costa Rica, El salvador, Guatemala, Honduras, Panamá; El caribe: Cuba, República Dominicana, Islas Guadalupe, Jamaica, Puerto Rico; Norte América: México; Suda-América: Argentina, Bolivia y Brasil, Colombia, Ecuador, Guayana francesa, Paraguay, Perú, Surinam, Trinidad, Venezuela; Para México se registran en Campeche, Chiapas, Coahuila, Guerrero, Jalisco, Michoacán, Morelos, Nayarit, Nuevo León, Oaxaca, Querétaro, San Luis Potosí, Tabasco, Tamaulipas, Veracruz (Atkinson, 2019).

Rango altitudinal. 1480 a los $2200 \mathrm{msnm}$.

Tipo de vegetación. Selva baja caducifolia, selva alta perennifolia.

Habito. Xilomicetófago.

Especificidad. Polífago.

Material examinado.- México; Morelos, TEPOZTLÁN: Sta. Catarina, 1550 m, S-52; 1720 m, Bursera copallifera, S-536; Amatlan, 1780 m, Bursera sp., SM-331; CUERNAVACA: 1850 m, Delonix regia; 1450 m, 1610 m,
Ficus sp., S-522; 1710 m, Ficus lyrata S-543; 1,450 m, Ficus retusa nitida, AB-34 (2); $1480 \mathrm{~m}$, Mangifera indica, AB-59; 1417 m, M. indica, AB-62 (2); 1670 m, Ficus sp., SM-64; 1450 m, AB-78; 1520 m, Ficus elastica SM-146; Cerro de la Herradura, 1720 m, AB-93 (2); YAUTEPEC: Fraxinus uhdei (1); Cañón de Lobos, 1460 m, Ficus cotinifolia, SM-207; 1640 m, AB-179; CUAUTLA: Cuautlixco SM-212; OCUITUCO: Jumiltepec, 2200 m, SM-311.

Hospederos. Anacardiaceae: Mangifera indica; Burseraceae: Bursera copallifera, Bursera sp.; Fabaceae: Delonix regia; Lonchocarpus macrophyllus; Juglandaceae: Carya illinoensis; Juglans sp.; Lauraceae: Persea sp.; Malvaceae: Heliocarpus appendiculatus, Heliocarpus sp., Teobroma cacao; Meliaceae: Cedrela fissilis; Moraceae: Ficus cotinifolia, Ficus elástica, Ficus lyrata, Ficus retusa filosa, Ficus sp.; Oleaceae: Fraxinus uhdei; Polygonaceae: Gymnopodium floribundum; Sapotaceae: Pouteria neglecta (Atkinson, 2019).

\section{Euplatypus parallelus (Fabricius 1801)} (Figuras 11-12)

Euplatypus parallelus (F., 1801), Systema Eleuth. 2: 324 Platypus parallelus (F., 1801), Systema Eleuth. 2: 324 Platypus poeyi (GuerinMeneville, 1825), Icon. Regne Animal 3: Bostrichus parallelus (F., 1801), Systema Eleuth. 2: 384

Platypus linearis (Stephens, 1830), Illust. Brit. Ent. 3: 419 Platypus praeustus (Dejean, 1837), Cat. Coleop. Coll. Dejean: Platypus testaceus (Dejean, 1837), Cat. Coleop. Coll. Dejean: 333 Platypus subcostatus (Jacquelin Duval, 1857), Hist. Phys. Polit. Nat. de l'ile Cuba: 238

Platypus compressus (Chapuis, 1865), Monographie des Platypides: 191 Platypus dejeani (Chapuis, 1865), Monographie des Platypides: 186 Platypus difficilis (Chapuis, 1865), Monographie des Platypides: 204 Platypus emarginatus (Chapuis, 1865) Monographie des Platypides: 199 Platypus erichsoni (Chapuis, 1865), Monographie des Platypides: 211 Platypus kraatzi (Chapuis, 1865), Monographie des Platypides: 196 Platypus laevicollis (Chapuis, 1865), Monographie des Platypides: 212 Platypus lebasi (Chapuis, 1865), Monographie des Platypides: 197 Platypus maeklini (Chapuis, 1865), Monographie des Platypides: 207 Platypus marseuli (Chapuis, 1865), Monographie des Platypides: 188 Platypus oblongus (Chapuis, 1865), Monographie des Platypides: 293 Platypus praevius (Chapuis, 1865), Monographie des Platypides: 205 Platypus proximus (Chapuis, 1865), Monographie des Platypides: 188 Platypus punctulatus (Chapuis, 1865), Monographie des Platypides: 199 Platypus regularis (Chapuis, 1865), Monographie des Platypides: 192 Platypus reticulatus (Chapuis, 1865), Monographie des Platypides: 194 Platypus rotundatus (Chapuis, 1865), Monographie des Platypides: 195 Platypus rugulosus (Chapuis, 1865), Monographie des Platypides: 192 Platypus subaequalis (Chapuis, 1865), Monographie des Platypides: 200 Platypus wesmaeli (Chapuis, 1865), Monographie des Platypides: 201 Platypus congoanus (Duvivier, 1891), Ann. Soc. Ent. Belq. 35: 363 Platypus triquetus (Brethes, 1909), An. Mus. Argentino Bernardo Rivadivia 3(19): 226

Platypus mattai (Brethes, 1919), Rev. Chilena Hist. Nat. Pura Aplicada 23: 26

Platypus bellus (Schedl, 1933), Rev. d' Ent. 3: 172

Distribución. África: Angola, Camerún, Costa de Marfil, Guinea Ecuatorial, Fernando Poo, Gabón, Ghana, Guinea, Kenia, Liberia, Madagascar, Nigeria, República de Sudáfrica, Santo Tomé y Príncipe, Senegal, Sierra Leona, Tanzania, Togo, Uganda, Zaire; Asia: Camboya, India, Tailandia. América Central: Belice, Costa Rica, El Salvador, Guatemala, Honduras, Nicaragua, Panamá; El Caribe: Bahamas, Islas Caimán, Cuba, República Dominicana, Gua- 
dalupe, Haití, Jamaica, Puerto Rico, Sta. Lucia, Islas Vírgenes, Europa: Inglaterra; Norte América: Estados Unidos y México; América del sur: Argentina, Bolivia, Brasil, Chile, Colombia, Ecuador, Guayana Francesa, Guyana, Paraguay, Perú, Surinam, Trinidad, Uruguay y Venezuela; Oceanía: Australia. De amplia distribución en México: Baja California, Campeche, Chihuahua, Chiapas, Guerrero, Jalisco, Morelos, Nayarit, Nuevo León, Oaxaca, Quintana Roo, Sinaloa, San Luis Potosí, Sonora, Tabasco, Tamaulipas, Veracruz y Yucatán (Atkinson, 2019).

Rango altitudinal. 920-1740 msnm.

Tipo de vegetación. Selva Baja Caducifolia, Selva alta perennifolia, matorral xerófilo y bajo espinoso.

Hábito. Xilomicetófago.

Especificidad. Polífago.

Material examinado. México; Morelos, CUERNAVACA: Delonix regia (1); 1610 m, Ficus sp., 1526; $1740 \mathrm{~m}$, Mangifera indica, AB-62 (2); $1670 \mathrm{~m}, \mathrm{Fi}$ cus sp., SM-64 (1); 1540 m, AB-78; Cerro La Herradura, 1720 m, AB-93; YAUTEPEC: Fraxinus uhdei; Cañón de Lobos, 1720 m, Leguminosae, AB-155; (1330 m, Bursera sp., SM-176; 1380 m, Bursera sp., SM-194; 1380m, Ficus sp.,SM-195; 1380 m, SM-197; 1320 m, Ceiba aesculifolia, SM-200; 1360 m, Erythrina brevifolia, SM-202; 1460 m, Ficus cotinifolia, SM-207; 1300 m, Ficus sp., SM-234; 1480m, Ficus sp., SM-248; 1350 m, Ceiba aesculifolia SM299; 1370 m, SM-301; TEPOZTLÁN: Santiago Tepetlapa, 1660 m, SM-315; TEMIXCO: 1270 m, Ficus sp., S-318 (1); 1270 m, Taxodium mucronatum S-561 (1); VILLA DE AYALA: Las piedras, 1110 m, Mangifera indica (2); 1060 m, SM-97; Huitzililla, 1200 m, AM-111 (2); Anenecuilco, 1110 m, Ficus elastica, S-664; TLAQUILTENANGO: El Vergel, 920 m, Ficus sp., AB-123; Cruz Pintada, 03-021996, 1000 m, AB-973, (4); 03-02-1996; 1000 m, AB-967 (3); 12-04-1997, $1000 \mathrm{~m}, 18^{\circ} 27^{\prime} 50^{\prime \prime} \mathrm{N} 99^{\circ} 02^{\prime \prime} 11^{\prime \prime} \mathrm{W}$, Col. A. Burgos (1); PUENTE DE IXTLA: Carr. La Tigra-El Zapote, 18-08-2001, 1110 m, 18 90'41' 'N 99²7'98' W, Ceiba parvifolia, Col. A. Burgos (1).

Hospederos. Anacardiaceae: Astronium graveolens, Mangifera indica, Metopio brownei, Spondias purpurea; Apocynaceae: Aspidosperma megalocarpon; Arecaceae: Areca catechu, Cocos nucifera; Bignoniaceae: Tabebuia sp.; Burseraceae: Bursera sp.; Euphorbiaceae: Croton nitens, C. pseudoniveus; Fabaceae: Acacia dolychostachya, Acacia guameri, Acrocarpus sp., Delonix regia, Erythrina brevifolia, Lonchocarpus rugoso, Lonchocarpus sp., Lesiona bahamensis, Pterocarpus rohrii; Malváceas: Ceiba aesculifolia, C. sp.; Meliaceae: Carapa slateri; Moraceae: Brosimum alicastrum; Cecropia obtusifolia, Ficus cotinifolia, Ficus elastica, Ficus radulina, Ficus retusa, Ficus sp.; Nyctaginaceae: Guapira sp. ; Oleaceae: Fraxinus uhdei; Pinaceae: Pinus oocarpa; Polygonaceae: Gymnopodium floribundum; Sapindaceae: Koelreuteria formanosa, Thouinidium decandrum; Taxodiaceae: Taxodium mucronatum; Ulmaceae: Celtis laevigata; Verbenaceae: Gmelina arbórea, Vitex guamerí (Atkinson, 2019).

Observaciones. Es la especie de Euplatypus más común encontrada en áreas tropicales de Morelos. Por lo regular infesta rama y árboles derribados o en pie, de diámetro mayor a $30 \mathrm{~cm}$. Sus galerías las establece en los lados de tronco y por debajo, con el propósito de evitar la incidencia de los rayos solares. La forma de la galería es irregular, sin un patrón definido y localizado en el xilema y de aproximadamente entre 15 a $25 \mathrm{~cm}$ de longitud. Especie muy común en lugares abiertos y perturbados, en las partes bajas de la entidad. Esta especie entra en competencia con otros grupos de escarabajos barrenadores, entre los que destacan cerambícidos, curculiónidos, bréntidos y Xyleborus volvulus. Esta especie es altamente polífaga ya que infesta a un buen número de plantas no emparentadas filogenéticamente. Esta especie es la más común en regiones tropicales y subtropicales del país.

\section{Euplatypus pini (Hopkins, 1905)}

(Figuras. 13-14)

Euplatypus pini (Hopkins, 1906) Proc. Ent. Soc. Wash. 7: 71 Platypus pini (Hopkins, 1906) Proc. Ent. Soc. Wash. 7: 71 Platypus quadridens (Schedl) 1937 Ent. Blatt. 33: 38

Distribución. América Central: Guatemala, Honduras, Nicaragua; Norte América: Estados Unidos: México: en el país se ha colectado en Chiapas, Durango, Hidalgo, México, Michoacán, Morelos, Nuevo León, Puebla, San Luis Potosí, Tlaxcala y Veracruz (Atkinson, 2019).

Rango altitudinal. 1900 a los 2660 msnm.

Tipo de vegetación. Bosque de pino-encino, bosque de pino.

Hábito. Xilomicetófago.

Especificidad. Monófago.

Material examinado. México; Morelos, HUITZILAC: Coajomulco, Pinus sp. (1); al sur de Coajumulco 2470 m, Pinus sp, SM-123(2); 2440 m, Pinus sp., S-539; Huitzilac, 2600, Pinus sp. (2); 2490, Pinus sp., S-552; El Tepeite, 2690 m, Pinus sp., S-535; 2660 m, Pinus sp., S-598; El Capulín, 2950 m, Pinus sp., S-646 (2); Fierro de Toro, 2990 m, Pinus sp., AB-28; Tlacotepec, 2600 m, Pinus sp., SM-320; CUERNAVACA: Chamilpa, 1900 m, Pinus montezumae, S-506(2), 503; TLANEPANTLA: Carr. Xochimilco-Oaxtepec, km. 56, 2500 m, Pinus sp., AB49(2); TETELA DEL VOLCÁN: Tlalmimilulpan, $2350 \mathrm{~m}$, Pinus sp., SM-307.

Hospederos. Pinaceae: Pinus chihuahuana, P. leiophylla, P. montezumae, P. ochoterenai, P. oocarpa, P. patula, P. ponderosa, P. pseudostrobus, $P$. rudis, $P$. sp. (Atkinson, 2019).

Observaciones. Esta especie se limita a arboles del género Pinus, talados o muertos en pie. No se presenta en pinares arriba de los $3000 \mathrm{msnm}$, por lo general limita sus ataques a partes del huésped con diámetro mayor de $30 \mathrm{~cm}$.

\section{Euplatypus longius (Wood, 1966)}

(Figuras 15-16)

Euplatypus longius (Wood, 1966) Great Basin Nat. 26 (3-4):57

Platypus longius (Wood, 1966) Great Basin Nat. 26 (3-4):57

Distribución. América Central: Guatemala, Honduras; Norte América. En México se ha registrado en Guerrero, 
México y Morelos (Atkinson, 2019).

Rango altitudinal. 2109 a los $2610 \mathrm{msnm}$.

Tipo de vegetación. Bosque de pino-encino, bosque de pino.

Hábito. Xilomicetófago.

Especificidad. Polífago.

Material examinado. México; Morelos, HUITZILAC: EI Tepeite, $2460 \mathrm{~m}$; TEPOZTLÁN: curva la pera, 2190 m, Lauraceae, 8-670(2); 2190 m, Clethra mexicana, S-669(2); San Juan Tlacotenco, 2190 m, Lauraceae, S-678; Sto. Domingo Ocotitlán, 2370 m, Clenthra mexicana, S-690; 2470 m, Alnus firmifolia, S-669(2); 2370 m, A. firmifolia, S-668(2); CUERNAVACA: 2109 m, Fraxinus uhdei, S-350(1); TÉTELA DEL VOLCÁN: las Cruces $2610 \mathrm{~m}$, Alnus sp., AB-207.

Hospederos. Betulaceae: Alnus firmifolia, Alnus acuminata, Alnus sp., Clethraceae: Clethra mexicana, Fagaceae: Quercus tomentocaulis; Lauraceae; Oleaceae: Fraxinus uhdei (Atkinson, 2019).

Observaciones. Especie encontrada en troncos de un diámetro mayor a $40 \mathrm{~cm}$. Es frecuente encontrarla en las barrancas, arroyos.

\section{Euplatypus otiosus (Schedl, 1936)}

(Figuras 17-18)

Euplatypus otiosus (Schedl 1936) Arch. Inst. Biol. Veg. Río de Janeiro 3: 100

Platypus otiosus (Schedl 1936) Arch. Inst. Biol. Veg. Río de Janeiro 3: 100

Distribución. Norte América: México. Para México se tienen registro en Durango, Hidalgo, Estado de México, Morelos, Tlaxcala y Oaxaca (Atkinson, 2019).

Rango altitudinal. 2380 a los $2470 \mathrm{msnm}$.

Tipo de vegetación. Bosque de pino-encino, Mesófilo.

Hábito. Xilomicetófago.

Especificidad. Polífago.

Material examinado. México; Morelos, HUITZILAC: Coajomulco, $\mathbf{2 4 7 0}$ m, Quercus sp., S-541; TEPOZTLAN: Sto. Domingo Ocotitlán, $2380 \mathrm{~m}$, Alnus acuminata, S-688; 2430 m, Quercus sp., S-695.

Hospederos. Betulaceae: Alnus acuminata, Alnus jorulensis, Fagaceae: Quercus sp., Quercus rugosa (Atkinson, 2019).

Observaciones. En comparación con E. longius parece estar limitado a altitudes menores. En la región de San José de los Laureles, se realizó una colecta de esta especie en árboles en pie de aproximadamente 40 a $50 \mathrm{~cm}$ de diámetro, aparentemente sanos.

\section{Distribución de las especies por tipo de vegetación}

Al analizar la información y actualizar los datos, nos permiten obtener un patrón más claro sobre la distribución de los platipodínos por tipo de vegetación. La selva baja caducifolia alberga más del $50 \%$ de las especies de platipodinos, seguida de bosque de Pinus-Quercus, Mesófilo, Agricultura perenne y ornamental con un $25 \%$ para cada tipo de vegetación y Bosque de Abies-Pinus está representado con una sola especie con un $12.5 \%$. Cabe aclarar que en algunos casos existen especies que pueden traslaparse de un tipo de vegetación a otra, por lo que el porcentaje varía. (Cuadro 2).

\section{CONCLUSIONES}

1. En este estudio se analiza un periodo de tiempo de más de 50 años, sobre el estudio de estos barrenadores en el estado de Morelos, lo que significa que el estado de conocimiento faunístico del grupo es aparentemente suficiente, sin embargo es posible que pueda al parecer unas dos o tres especies en regiones aún no exploradas en la entidad tal es el caso de especies colectadas en la región de Mexicapa en el estado de México asociado a barrancas cuya vegetación se caracteriza por presentar elementos mesófilos.

2. Se revisaron y analizaron siete especies de platipodinos registrados para el estado de Morelos.

3. Oxoplatypus quadridentatus constituye un nuevo registro para la entidad, siendo el registro más sureño; además se presenta un nuevo hospedero Quercus magnoliifolia Née.

4. Se adiciona información sobre nuevas localidades de las especies, lo que actualiza su rango de distribución para la entidad.

5. Se registraron cuatro nuevos hospederos para las siguientes especies Schedlarius mexicanus asociado a Bursera fagaroides (Burseraceae), Megaplatypus exaratus a Erythrina aff. breviflora, (Fabaceae) Euplatypus parallelus a Ceiba parvifolia (Bombacaceae) y Teloplatypus ustulatus a Lysiloma sp. (Mimosaceae).

\section{AGRADECIMIENTOS}

Los autores agradecemos los comentarios y sugerencias al escrito, asimismo agradecemos a Edgar Martínez y Eduardo Saucedo por permitir incluir sus colectas, sin ellos no hubiese sido posible la culminación de este trabajo. Finalmente, este documento forma parte de las actividades del Cuerpo Académico de Entomología y Fitopatología UAEM-CA-073, así como el proyecto UAEM, PII-69 "Establecimiento de una colección de Referencia (CEUM), del Centro de Investigaciones Biológicas-UAEMor. Al Dr. Thomas H. Atkinson de Texas Natural History Collection, Entomology y a la Biol. Patricia Jaimez del Laboratorio de Entomología del CIB. Finalmente agradecemos a los revisores del escrito por sus comentarios y sugerencias al documento.

\section{LITERATURA CITADA}

Aguilar B.S. 1990. Dimensiones ecológicas del estado de Morelos. Centro Regional de Investigaciones Multidisciplinarias, UNAM.

Alonso, R., A. de Errastri, D. Gómez, C. Pérez, L. Bettucci, M. Rajchenberg y M. M. Pildaín 2014. Ophiostomatales asociados a escolítidos en plantaciones de pino en Uruguay. https://www.researchgate.net/publication/281649806_Ophiostomatales_asociados_a_escolitidos_en_plantaciones_de_pino_en_Uruguay [accessed Jun 24 2019]. 
Alvidrez-Villarreal, R., F.D. Hernández-Castillo, O. García-Martínez, R. Mendoza-Villarreal, R. Rodríguez-Herrera, C.N. Aguilar-González 2010. Metabolitos Secundarios en Tejido de Nogal Pecanero Dañado por el Barrenador Ambrosial (Euplatypus segnis Chapuis) y Hongos Asociados. Revista Agraria -Nueva EpocaAño VII Vol. 7 Nos. 1, 2, 3.

Anónimo, 1981. Síntesis Geográfica de Morelos. Secretaría de Programación y Presupuesto.

Atkinson, T. H. 2019. Bark and Ambrosial beetles. http:// www.barkbeetles.info/articles.php

Atkinson, T.H., E. Saucedo Céspedes, E. Martínez Fernández, y A. Burgos Solorio. 1986a. Coleópteros Scolytidae y Platypodidae asociados con las comunidades vegetales del clima templado frío en el Estado de Morelos, México. Acta Zoológica Mexicana. 17: 12-13.

Atkinson, T. H., E. Martínez-Fernández, E. Saucedo-Cespedes, y A. Burgos-Solorio. 1986b. Los Scolytidae y Platypodidae (Coleóptera). asociados a Selva baja caducifolia y comunidades derivadas al Estado de Morelos. Folia Entomológica Mexicana 69: 41-82.

Atkinson, T.H. y A. Equihua M. 1985. Lista comentada de los coleópteros Scolytidae y Platypodidae del Valle de México. Folia Entomológica Mexicana 65: 63-108.

Atkinson, T.H. and A. Equihua M. 1986. Biology of Scolytidae and Platypodidae (Coleoptera) of a tropical rain forest in southeastern Mexico with an annotated checklist of species. Annles of Entomolgical Society of America 79: 414-423.

Batra, L.R. 1966. Ambrosia fungi: extent of specificity to ambrosia beetles. Science, 153, 193-195.

Batra, L.R., 1985. - Ambrosia beetle and their associated fungi: Research trends and techniques. Proceedings of the Indian Academy of Sciences 49: 137-148.

Blackman, M. W. 1922. Mississippi Bark Beetles. Technical Bulletin No. 11 1-130

Burgos, S. A. y A. G. Trejo-Loyo. 2001. Lista preliminar de los Coleópteros registrados para el Estado de Morelos, México. En: Navarrete-Heredia, J. L., H. E. Fierros-López y A. Burgos-Solorio (Eds). Tópicos sobre Coleóptera de México. Universidad Autónoma de Guadalajara y Universidad Autónoma del Estado de Morelos.

Burgos, S.A. y A. Equihua M. 2007. Platypodidae y Scolytidae (Coleóptero) de Jalisco, México. Dugesiana 14(2): 58-82.

Burgos, S.A. y T. H. Atkinson. 2015. Redescripción de Megaplatypus exaratus (Blandford 1896 notas sobre su distribución en México y Centroamérica (Coleoptera: Curculionidae: Platypodinae) Insecta Mundi 1-10
Dorado, R. O. R. 1997. Inventario Florístico de la Sierra de Huautla zonas adyacentes del Estado de Guerrero y Puebla. Reporte de CONABIO.

Equihua M, A.; T.H. Atkinson, y E.J. Lott. 1984. Scolytidae y Platypodidae (Coleoptera) de la Estación de Biología Chamela, Jalisco. Agrociencia 57: 179-193.

Equihua, M. A. 1985. Nuevos registros de localidades de huéspedes de coleópteros Platypodidae americanos. Folia Entomológica Mexicana 66: 143-144.

Equihua, M. A. y T. H. Atkinson 1987. Catálogo de Platypodidae (Coleoptera) de Norte y Centroamérica. Folia Entomológica Mexicana, (72): 5-31.

Eskalen A., J. Kabashima and M. Dimson. 2014. Polyphagous Shot Hole Borer + Fusarium Dieback Field Identification Guide. University of California. Agricultura and Natural Resources, California, United Estates.

Freeman S., M. Sharon, M. Maymon, Z. Mendel, A. Protasov, T. Aoki, A. Eskalen and K. O'Donnell. 2013. Fusarium euwallaceae sp. nov.-a symbiotic fungus of Euwallacea sp., an invasive ambrosia beetle in Israel and California. Mycologia. Nov-Dec; 105(6):1595-1606. doi: 10.3852/13-066.

Martínez, F. E. 1984. Los escarabajos descortezadores y ambrosiales (Coleoptera: Scolytidae y Platypodidae) del Cañón de Lobos, Yautepec, Morelos. Tesis de Licenciatura. Universidad Autónoma del Estado de Morelos.

Noguera, M., F. A. and T.H. Atkinson. 1990. Biogeography and biology of bark and ambrosia beetles (Coleoptera: Scolytidae and Platypodidae) in a mesic montane forest in Mexico with an annotated checklist of species. Annles of Entomolgical Society of America 83(3): 453-466.

Samaniego-Gaxiola, J. A., M. Ramírez-Delgado, A. Pedroza-Sandoval y U. Nava-Camperos 2008. Asociación entre pudrición texana (Phymatotrichopsis omnivora) e insectos barrenadores del nogal (Carya illinoensis) Agricultura Técnica en México. 34(1) http://scielo. unam.mx/pdf/agritm/v34n1/v34n1a3.pdf

Schedl, K.E. 1940. Scolytidae, Coptonotidae y Platypodidae Mexicanos. Anales de la Escuela Nacional de Ciencias Naturales, IPN 1(3-4): 317-376.

Wood, S. L. 1982. The bark and ambrosia beetles of North and Central America (Coleoptera: Scolytidae), a Taxonomic Monograph. Great Basin Naturalist Memoirs, 6: $1-1359$.

Wood, S.L. 1992. Nomenclatural Changes in Scolytidae and Platypodidae (Coleoptera). Great Basin Naturalist 52 (1): 89-92.

Wood, S.L. 1993. Revision of the genera of Platypodidae (Coleoptera). Great Basin Naturalist 53 (3): 259-281.

Wood, S.L. and D.E. Bright. 1992. A catalog of Scolytidae and Platypodidae (Coleoptera), part 2. Taxonomic Index Volume A. Great Basin Naturalist Memories 13A.

Wood, S.L. and D.E. Bright. 1992. A catalog of Scolytidae and Platypodidae (Coleoptera), part 2. Taxonomic Index Volume B. Great Basin Naturalist Memories 13B 
Cuadro 1.- Resumen taxonómico de las especies de Platipodinos (Curculionidae: Platypodinae) para el estado de Morelos El arreglo taxonómico es acorde al criterio de Wood (1993) y Atkinson (2019).

\begin{tabular}{|l|l|l|}
\hline Tribu/Subtribu & Géneros & Especies \\
\hline $\begin{array}{l}\text { Schedlariini/ } \\
\text { Schedlariina }\end{array}$ & Schedlarius & Schedlarius mexicanus \\
\hline & Oxoplatypus & $\begin{array}{l}\text { Oxoplatypus quadriden- } \\
\text { tatus }\end{array}$ \\
\hline $\begin{array}{l}\text { Platypodini/ } \\
\text { Platypodina }\end{array}$ & Megaplatypus & Megaplatypus exaratus \\
\hline & Teloplatypus & Teloplatypus ustulatus \\
\hline & Euplatypus & Euplatypus segnis \\
\hline & & Euplatypus parallelus \\
\hline & & Euplatypus pini \\
\hline & & Euplatypus longius \\
\hline
\end{tabular}

Recibido: 25 de agosto 2019

Aceptado: 28 de octubre 2019
Cuadro 2.- Distribución de platipodinos por tipo de vegetación en el estado de Morelos

\begin{tabular}{|l|l|l|c|}
\hline $\begin{array}{c}\text { Tipo de ve- } \\
\text { getación }\end{array}$ & Altitud (m) & \multicolumn{1}{|c|}{ Especies } & $\%$ \\
\hline $\begin{array}{l}\text { Selva baja } \\
\text { Caducifolia }\end{array}$ & $900-1800$ & $\begin{array}{l}\text { Schedlarius mexi- } \\
\text { canus, Euplatypus } \\
\text { parallelus, Eupla- } \\
\text { typus segnis Telopla- } \\
\text { typus ustulatus }\end{array}$ & $50 \%$ \\
\hline $\begin{array}{l}\text { Bosque } \\
\text { Mesófilo de } \\
\text { montaña }\end{array}$ & $1900-2500$ & $\begin{array}{l}\text { Euplatypus otiosus } \\
\text { Euplatypus longius }\end{array}$ & $25 \%$ \\
\hline $\begin{array}{l}\text { Bosque de } \\
\text { Pinus-Quer- } \\
\text { cus }\end{array}$ & $2600-1800$ & $\begin{array}{l}\text { Euplatypus pini, } \\
\text { Euplatypus longius, } \\
\text { Euplatypus otiosus, } \\
\text { Oxoplatypus quadri- } \\
\text { dentatus }\end{array}$ & $25 \%$ \\
\hline $\begin{array}{l}\text { Agricultura } \\
\text { perenne y } \\
\text { ornamental }\end{array}$ & $1000-2500$ & $\begin{array}{l}\text { Euplatypus para- } \\
\text { llelus, Euplatypus } \\
\text { segnis }\end{array}$ & $25 \%$ \\
\hline $\begin{array}{l}\text { Bosque de } \\
\text { Abies-Pinus }\end{array}$ & $3000-2600$ & \begin{tabular}{l} 
Euplatypus pini \\
\hline
\end{tabular} & $12.5 \%$ \\
\hline
\end{tabular}



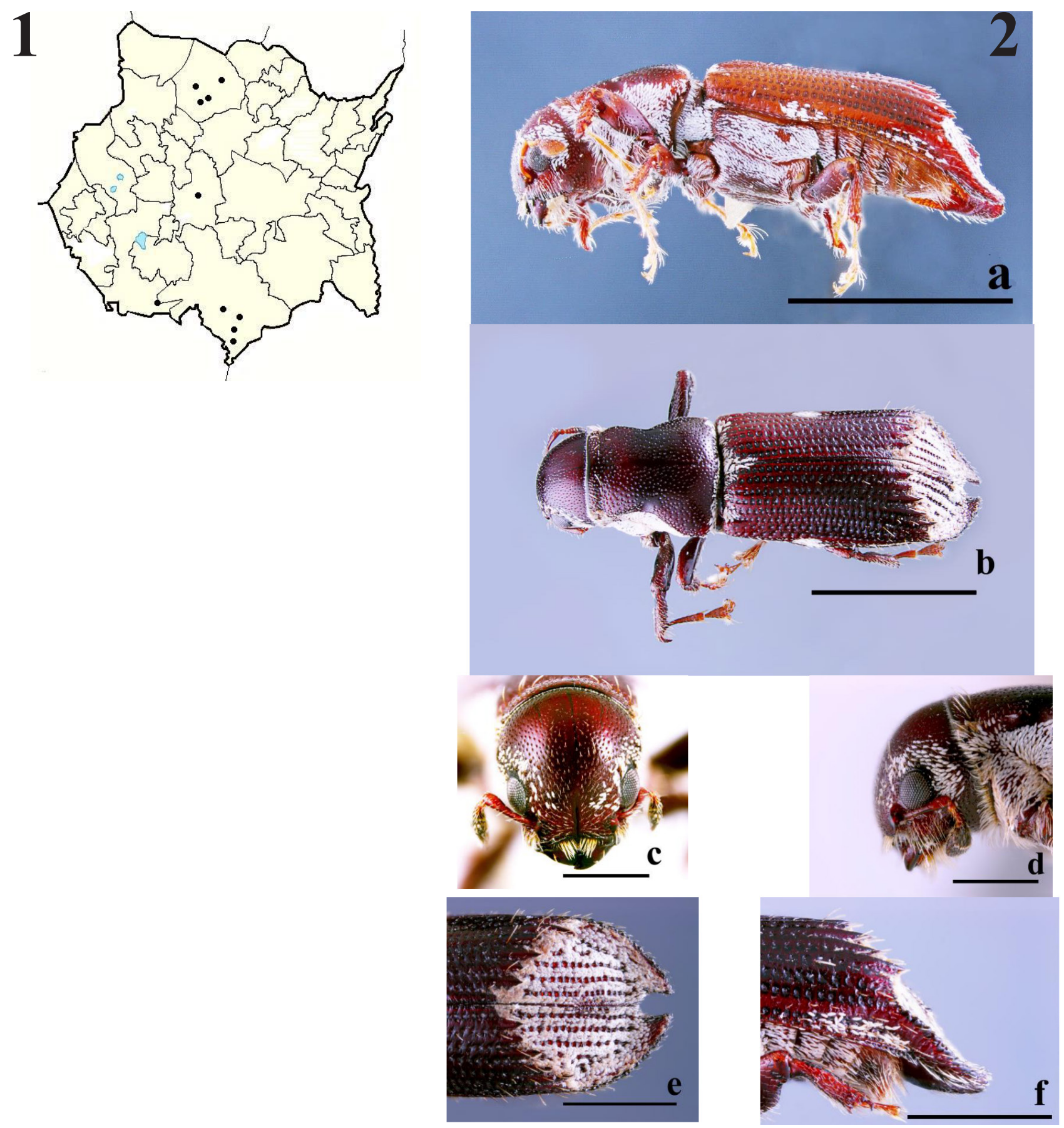

Figura 1.- Ubicación geográfica de Schedlarius mexicanus. Figura 2. a) Vista lateral de Schedlarius mexicanus, en la que se denota la quetotaxia; b) cabeza expuesta, Pronoto hendido declive elitral pronunciado caracteres de la especie; La línea representa $1 \mathrm{~mm}$; c) Vista frontal de la cabeza, en la que denota la línea media y prominentes de color negro; d) Vista lateral de declive de los élitros en la que señala las proyecciones agudas que delimitan el borde anterior de los élitros; e) Declive elitral delimitado por un borde ligeramente elevado y aserrado, cóncavo en sus extremos y ligeramente abultado en su parte central (en vista lateral), y ornamentados con escamas blancas, marcadas con cuatro hilera de puntos y borde posterior en forma de "U" con los ápices agudos f) Vista latera del declive elitral. La línea representa $0.5 \mathrm{~mm}$. 

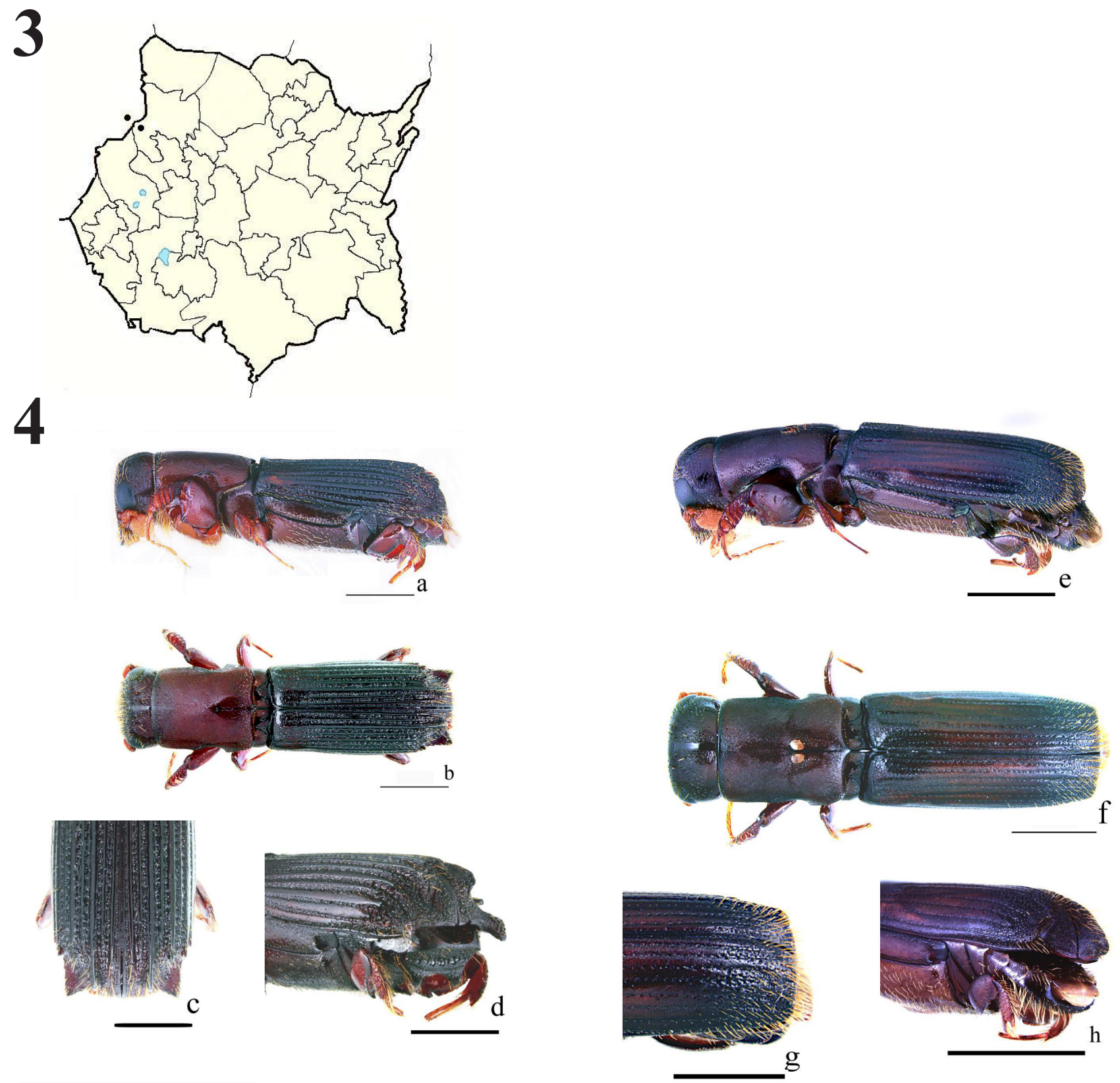

Figura 3. Distribución de Oxoplatypus quadridentatus en el estado de Morelos y Estado de México. Figura 4. a) Vista lateral del macho de la especie Oxoplatypus quadridentatus; b) Vista dorsal; c) Vista dorsal del declive elitral; d) Detalle de la parte posterior de los élitros del macho; e) Vista lateral de la hembra; f) Vista dorsal de la hembra; g) Vista dorsal del declive de la hembra; h) Vista lateroposterior del declive de los élitros de la hembra. La línea representa $1 \mathrm{~mm}$. 


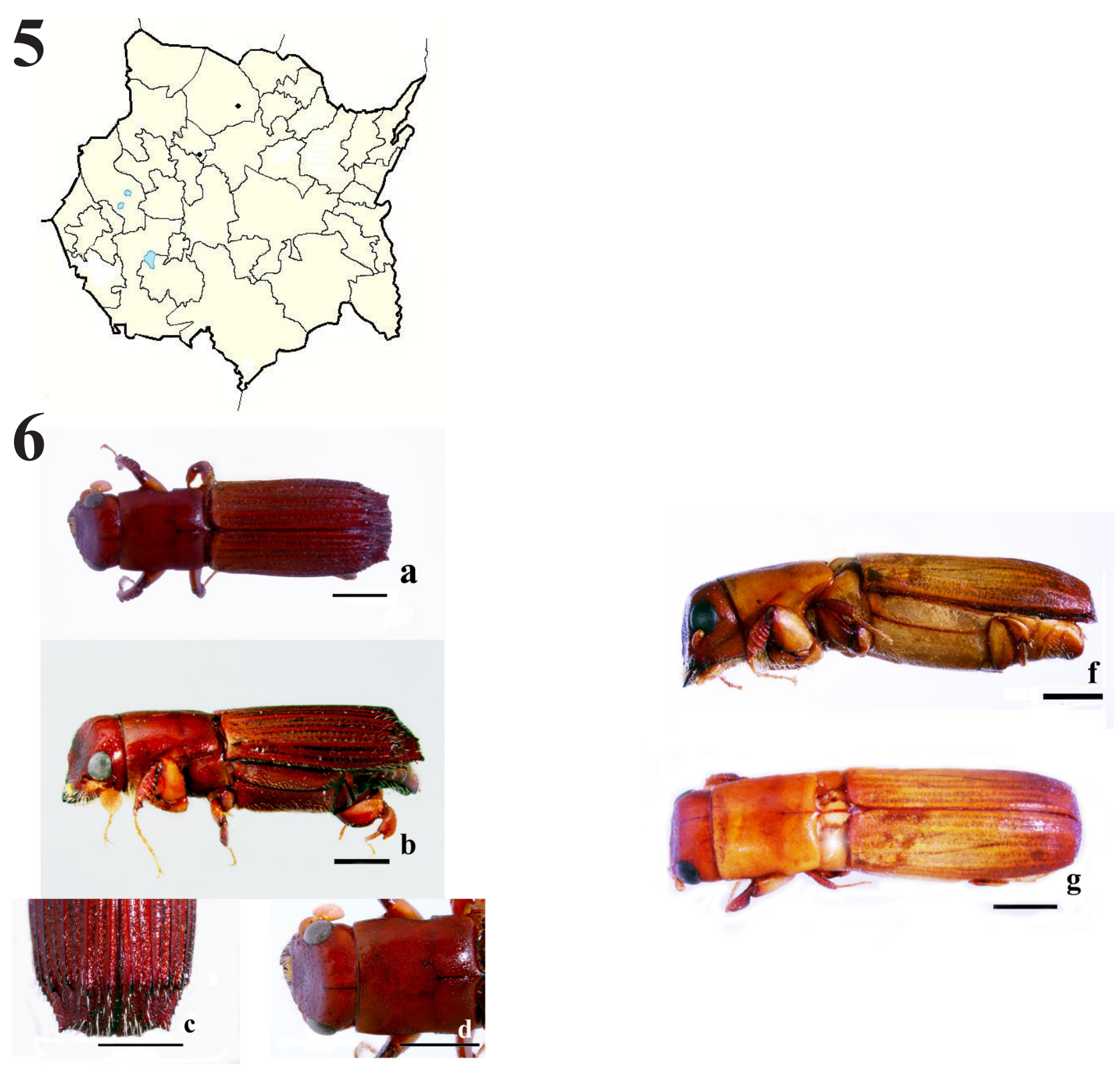

Figura 5.- Distribución de Megaplatypus exaratus en el estado de Morelos. Figura 6. a) Vista dorsal del macho de la especie Megaplatypus exaratus; b) Vista lateral; c) Vista dorsal del declive elitral el cual inicia a partir de donde terminan las hileras del élitro, se caracteriza por proyecciones agudas y oscuras; d) Detalle de Vista dorsal de la cabeza y pronoto; f) Vista lateral de la hembra; g) Vista dorsal de la hembra, élitros carece de estrías y proyecciones. En el borde posterior fuertemente angulado y el declive pronunciado. La línea representa $1 \mathrm{~mm}$. 


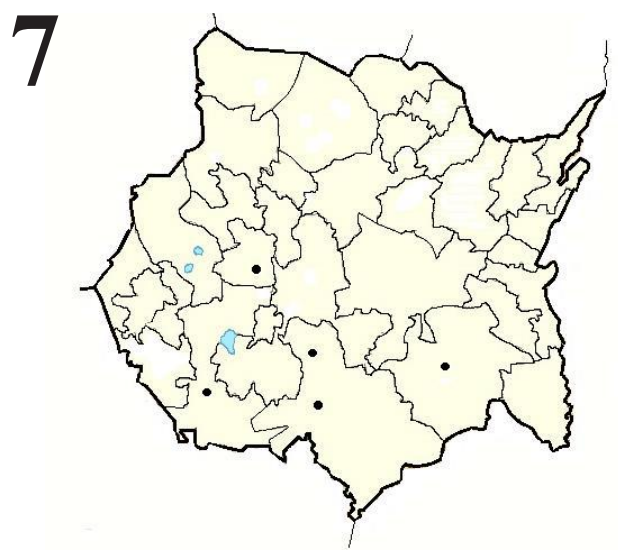

8
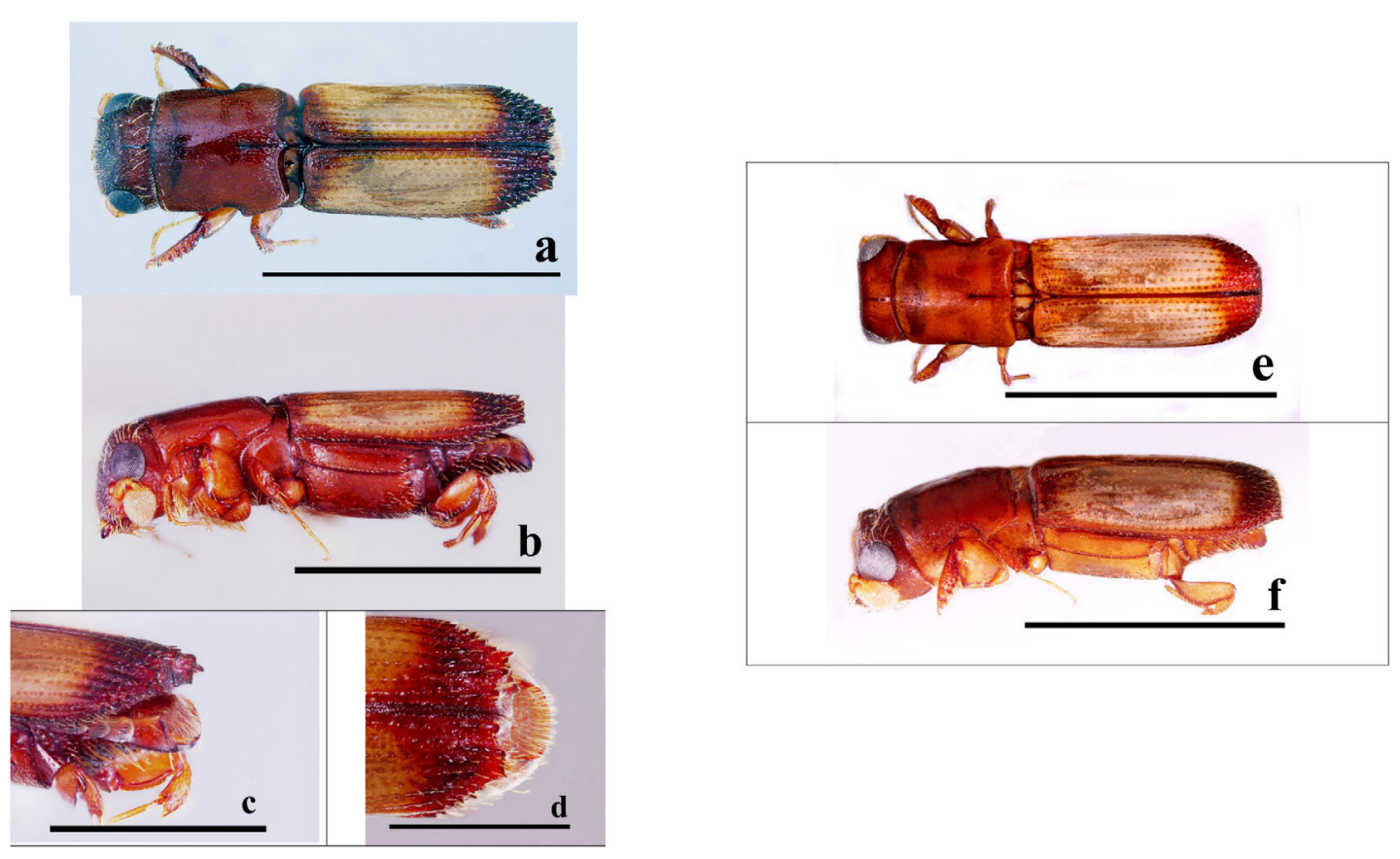

Figura 7.- Distribución de Teloplatypus ustulatus en el estado de Morelos. Figura 8. a) Vista dorsal del macho de Teloplatypus ustulatus, en la que se denota la cabeza, pronoto hendido, con una línea media en la región declive, elitral débilmente pronunciado con espinas cortas, borde laterales posteriores de los élitros aserrados; b) Vista lateral, cabeza expuesta, ojo prominentes, antena sin segmentos, posterior, pronoto hendido c) Detalle de las proyecciones en el declive del élitro cortos; d) vista lateral de élitro en la que denotan las proyecciones $\mathrm{n}$ dos niveles dos distribuida a los lados y seis corta sobre el declive. La línea representa $0.5 \mathrm{~mm}$; e) Vista lateral de la hembra en la que se distinguen una línea media del pronoto y f) Vista lateral en que se denotan los élitros truncados. La línea representa $1 \mathrm{~mm}$. 


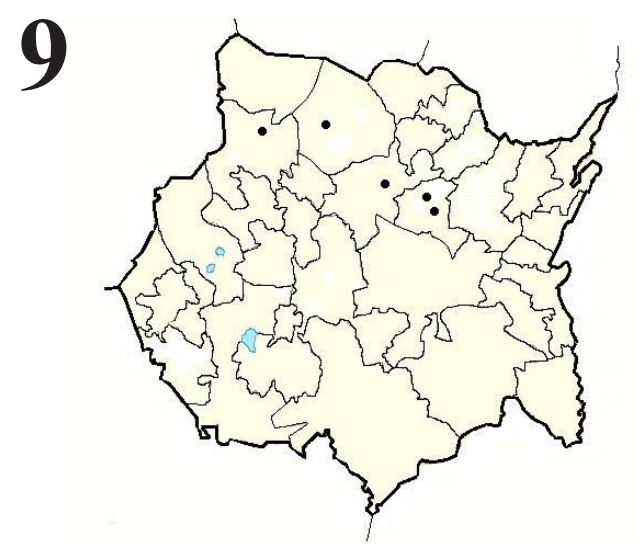

\section{0}
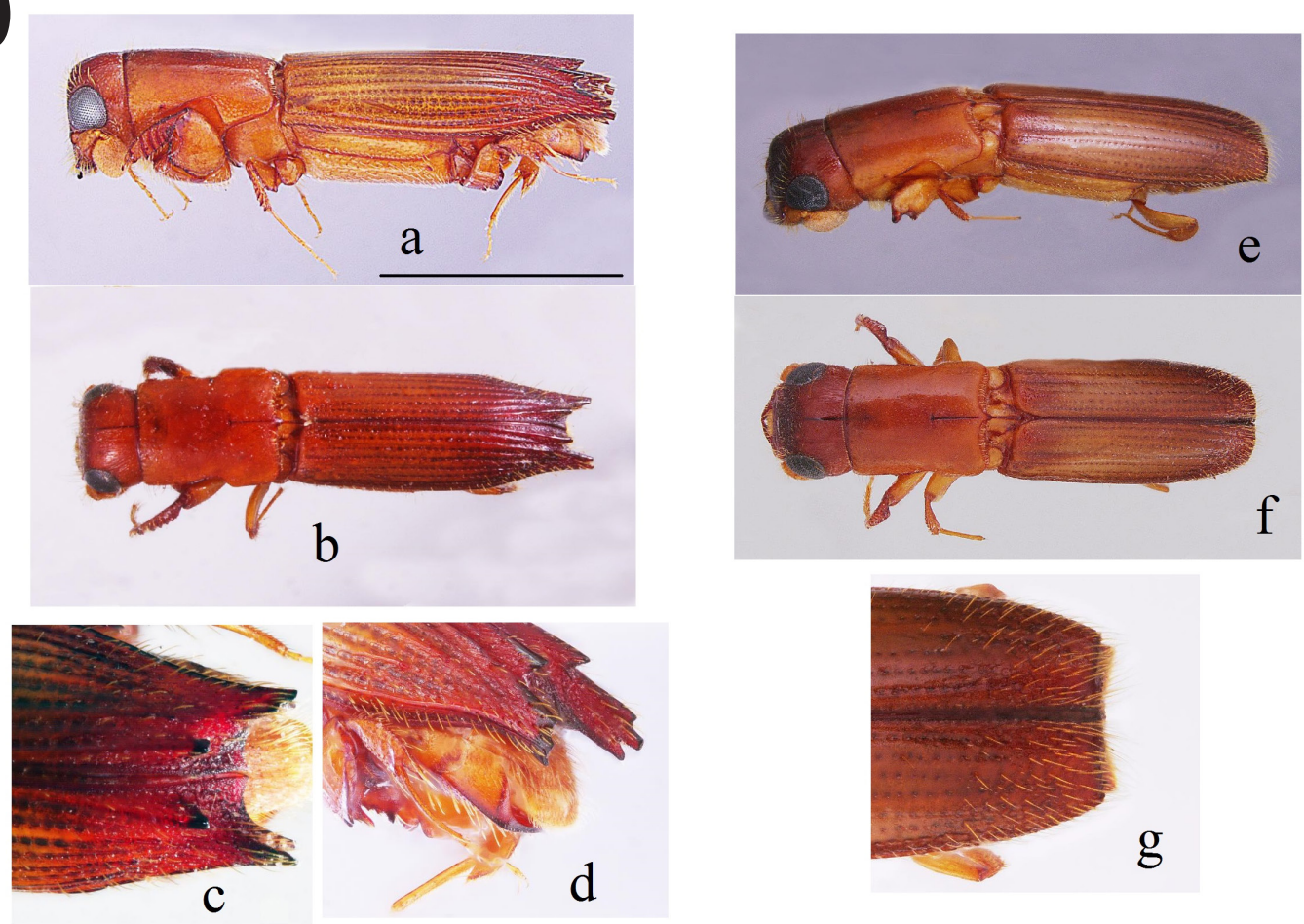

Figura 9.- Distribución de Euplatypus segnis en el estado de Morelos. Figura 10. a) Vista lateral de Euplatypus segnis en la que se distinguen las proyecciones del borde posterior, carácter distintivo de la especie. Las proyecciones que sobresalen de los élitros con ápices bífidos. La línea representa $1 \mathrm{~mm}$. b) Vista dorsal de Euplatypus segnis, se distinguen cuatro proyecciones, dos de ellas a los lados de la línea media de los élitros y las dos restantes, que se proyectan con los ápices bífidos. c) Vista dorsal del margen posterior. d) Detalle de las proyecciones del borde posterior. e) Vista lateral de la hembra de Euplatypus segnis con el borde posterior truncado. f) Vista dorsal de la hembra de Euplatypus segnis, cuya ornamentación con sedas doradas, las cuales se distribuyen en la región posterior de los élitros. g) Vista dorsal de borde posterior de la hembra de Euplatypus segnis con el borde truncado. 


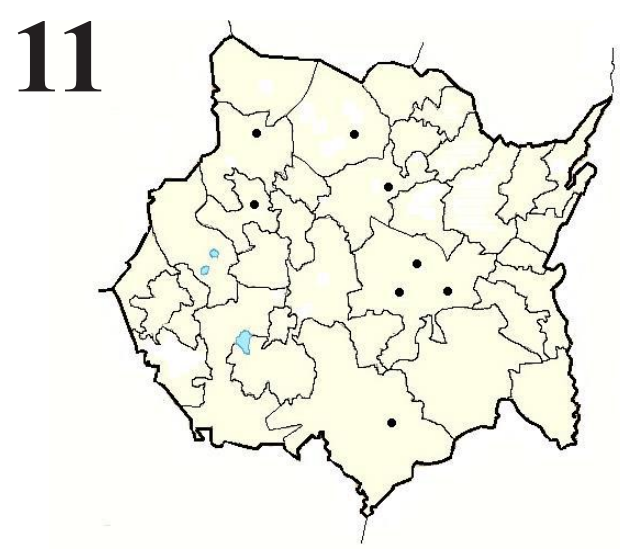

12
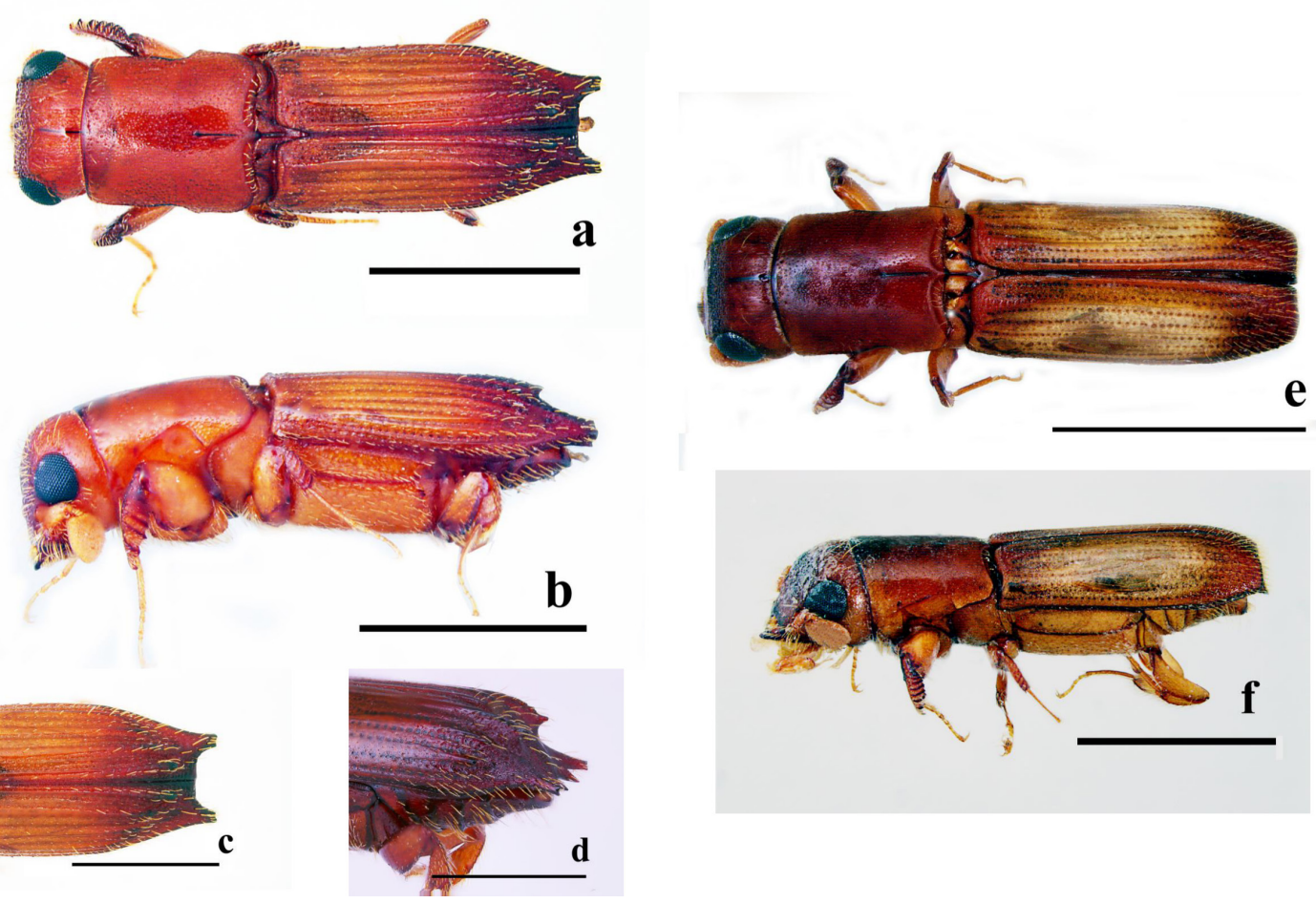

Figura 11.- Distribución de Euplatypus parallelus en el estado de Morelos. Figura 12. a) Vista dorsal del macho de Euplatypus parallelus, en la que se denota la quetotaxia, cabeza expuesta, Pronoto hendido declive elitral pronunciado caracteres de la especie; b) Vista lateral, cabeza expuesta, ojo prominentes, y antena sin segmentos; c) Detalle de las proyecciones en el declive del élitro con los ápices bífidos; d) Vista lateral de élitro en la que denotan las proyecciones; e) Vista dorsal de la hembra con el ápice élitral truncado y recto, f) vista lateral de la hembra, nótese los élitros truncados. La línea representa $1 \mathrm{~mm}$. 
13

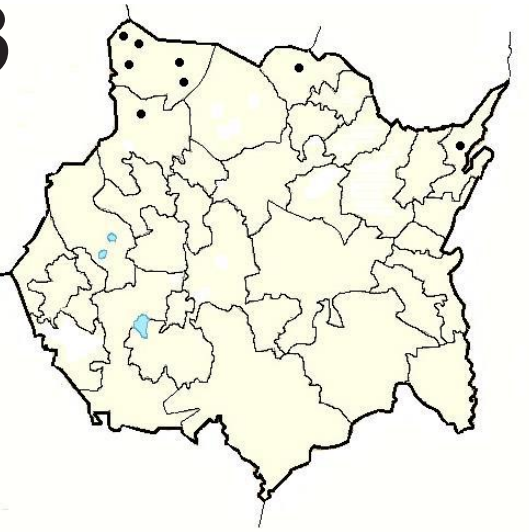

14
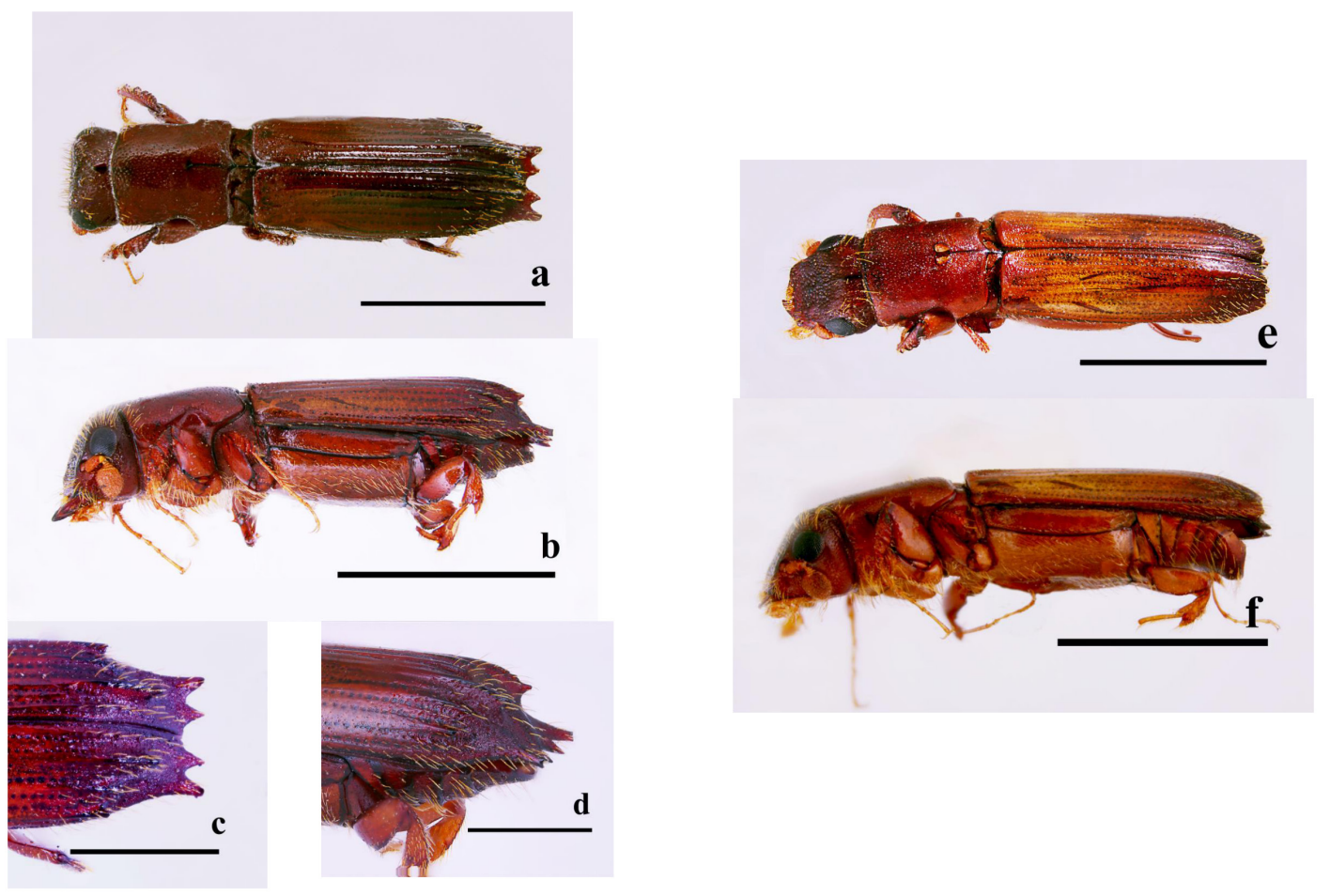

Figura 13.- Distribución de Euplatypus pini en el estado de Morelos. Figura 14. a) Vista dorsal del macho de Euplatypus pini, en la que se denota la cabeza, pronoto hendido declive elitral pronunciado caracteres de la especie; La línea representa $1 \mathrm{~mm}$ b) Vista lateral, cabeza expuesta, ojo prominentes, y antena sin segmentos; c) Detalle de las proyecciones en el declive del élitro en forma de "U" con los ápices ligeramente bífidos; d) vista lateral de élitro en la que denotan las proyecciones La línea representa $0.5 \mathrm{~mm}$; e) Vista dorsal de la hembra; f) Vista lateral. La línea representa $1 \mathrm{~mm}$. 


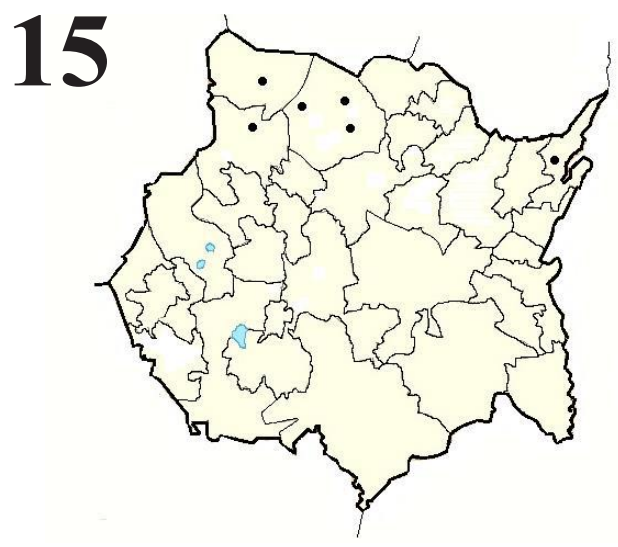

\section{6}
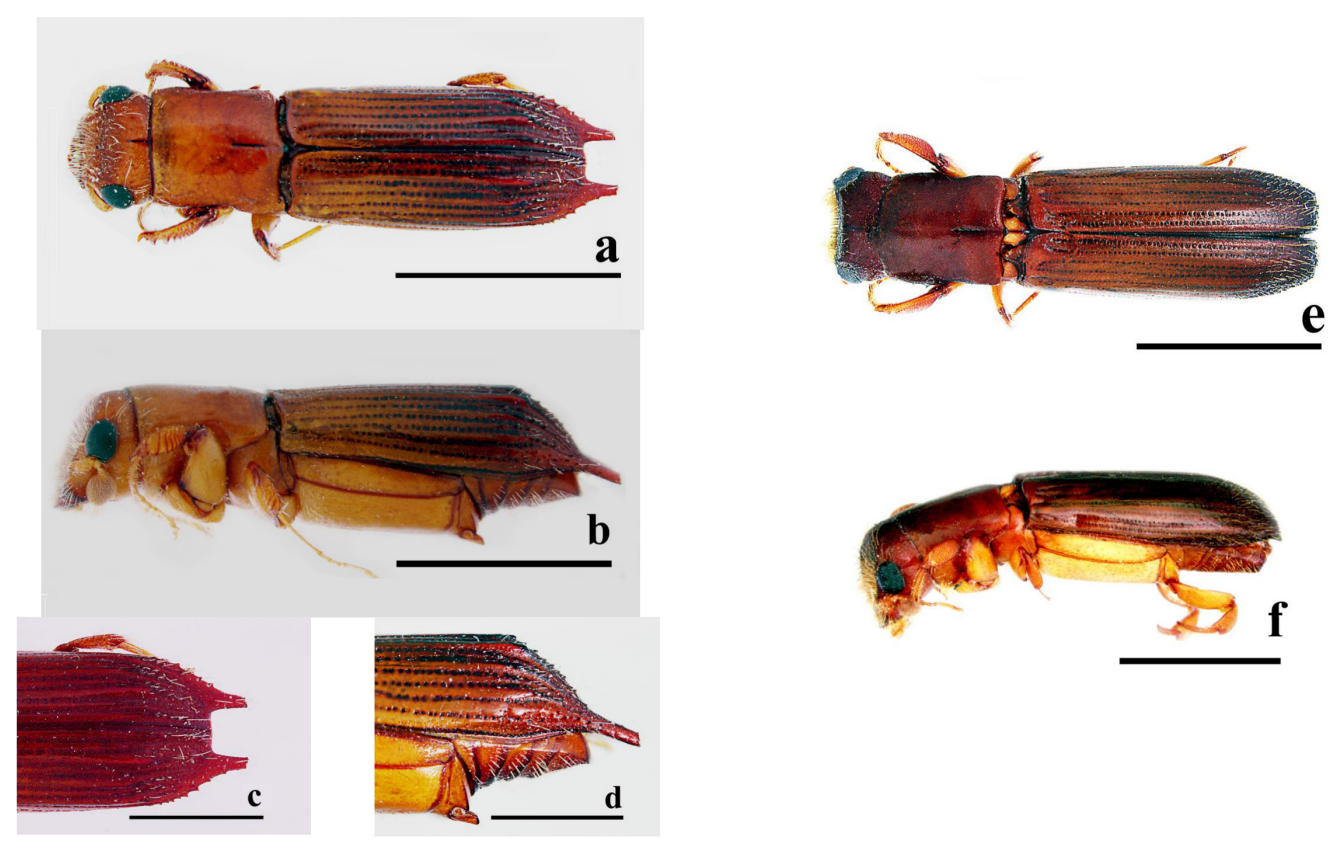

Figura 15.- Distribución de Euplatypus longius en el estado de Morelos. Figura 16. a) Vista dorsal del macho de Euplatypus longius, en la que se denota la cabeza, pronoto hendido declive elitral pronunciado caracteres de la especie; b) Vista lateral, cabeza expuesta, ojo prominentes, antena sin segmentos y declive y proyecciones de los élitros; c) Detalle de las proyecciones en el declive del élitro en forma de "U" con los ápices ligeramente bífidos; d) vista lateral de élitro en la que denotan las proyecciones La línea representa $0.5 \mathrm{~mm}$; e) Vista dorsal de la hembra en la que se distinguen los micangios sobre la parte media del pronoto y f) vista lateral en que se denotan los élitros truncados. La línea representa $1 \mathrm{~mm}$. 


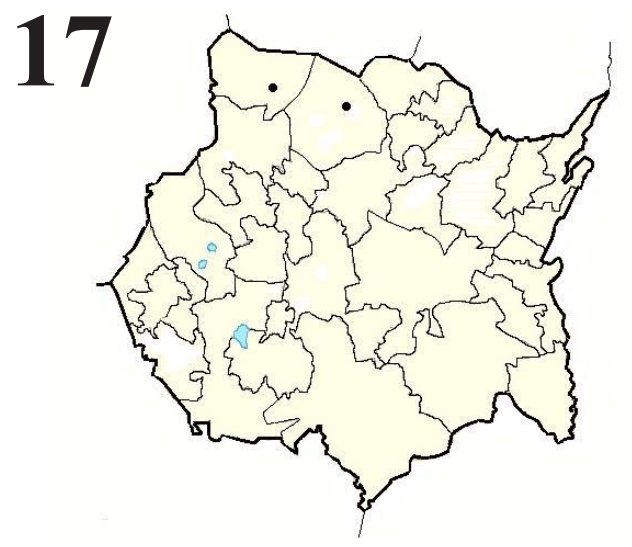

18
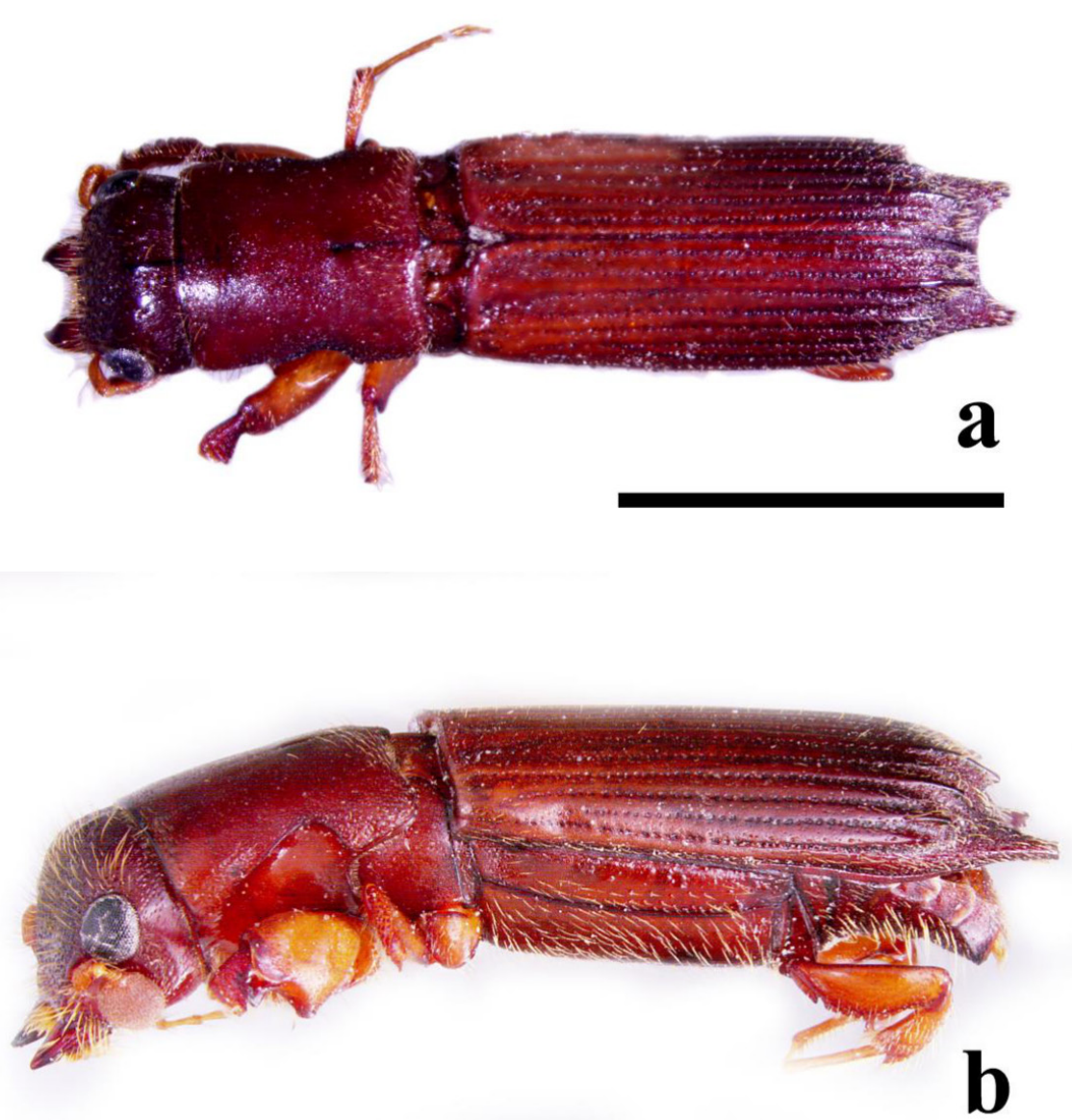

Figura 17.- Distribución de Euplatypus otiosus en el estado de Morelos. Figura 18. a) Vista dorsal del macho de Euplatypus otiosus, en la que se denota la cabeza, pronoto hendido declive elitral pronunciado caracteres de la especie; La línea representa $1 \mathrm{~mm}$ b) Vista lateral, cabeza expuesta, ojo prominentes, y antena sin segmentos; c) Detalle de las proyecciones en el declive del élitro en forma de "U" con los ápices ligeramente bífidos; d) vista lateral de élitro en la que denotan las proyecciones La línea representa $0.5 \mathrm{~mm}$ 